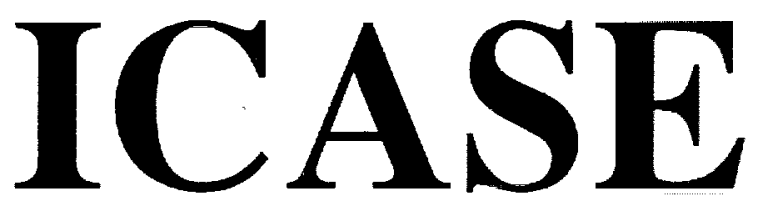

\title{
ON EXPLICIT ALGEBRAIC STRESS MODELS FOR COMPLEX TURBULENT FLOWS
}
T. B. Gatski
C. G. Speziale

NASA Contract Nos. NAS1-18605 and NAS1-19480

November 1992

Institute for Computer Applications in Science and Engineering NASA Langley Research Center

Hampton, Virginia 23681-0001

Operated by the Universities Space Research Association

\section{N/Sก}

National Aeronautics and Space Administration

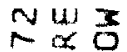

$0 \div \frac{1}{4}$

on

$\vec{x} \cup$

$x \stackrel{z}{0}$

1 a 


\title{
ON EXPLICIT ALGEBRAIC STRESS MODELS FOR COMPLEX TURBULENT FLOWS
}

\author{
T. B. Gatski \\ NASA Langley Research Center \\ Hampton, VA 23681 \\ C. G. Speziale* \\ ICASE, NASA Langley Research Center \\ Hampton, VA 23681
}

\begin{abstract}
Explicit algebraic stress models that are valid for three-dimensional turbulent flows in non-inertial frames are systematically derived from a hierarchy of second-order closure models. This represents a generalization of the model derived by Pope [J. Fluid Mech. 72, 331 (1975)] who based his analysis on the Launder, Reece and Rodi model restricted to twodimensional turbulent flows in an inertial frame. The relationship between the new models and traditional algebraic stress models - as well as anistropic eddy viscosity models - is theoretically established. The need for regularization is demonstrated in an effort to explain why traditional algebraic stress models have failed in complex flows. It is also shown that these explicit algebraic stress models can shed new light on what second-order closure models predict for the equilibrium states of homogencous turbulent flows and can serve as a useful alternative in practical computations.
\end{abstract}

*This research was supported by the National Aeronautics and Space Administration under NASA Contract Nos. NAS1-18605 and NAS1-19480 while the second author was in residence at the Institute for Computer Applications in Science and Engineering (ICASE), NASA Langley Research Center, Mampton, VA 23681 . 


\section{INTRODUCTION}

Turbulent flows contain length and time scales that can change dramatically from one flow configuration to the next. Consequently, two-equation turbulence models - wherein transport equations are solved for two turbulent fields that are directly related to the length and time scales - represent the simplest level of Reynolds stress closure that can be geometry independent. This explains the current popularity of the $K-\varepsilon$ model which, unlike the older mixing length models, does not require the specification of empirical length or time scales that must be adjusted in an ad hoc fashion from one flow to the next (see Launder and Spalding 1974). However, despite this positive feature, the $K-\varepsilon$ model shares a common deficiency with the older mixing length models: it is based on the Boussinesq eddy viscosity hypothesis. It is well-known that eddy viscosity models are unable to properly describe turbulent flows with body force effects arising from curvature or a system rotation. Likewise, their inaccurate prediction of normal Reynolds stress differences make eddy viscosity models incapable of describing secondary flows in non-circular ducts (c.f. Lumley 1978, Launder 1990 and Speziale 1991). This has led to the development of nonlinear stress-strain relationships in turbulence modeling that transcend the Boussinesq hypothesis - an area of research that has received varying degrees of attention during the past three decades.

Early work on the development of turbulence models with a nonlinear stress-strain relation tended to be empirical in nature, relying on analogies with non-Newtonian flows (see Rivlin 1957, Lumley 1970 and Saffman 1977). The similarities between the laminar flow of a non-Newtonian fluid and the mean turbulent flow of a Newtonian fluid have long been recognized (see Hinze 1975 for an interesting discussion of this point). In these older empirical models, which typically were obtained by simple tensor invariance arguments, the Reynolds stresses were taken to be nonlinear polynomial functions of the mean velocity gradients. In recent years, nonlinear Reynolds stress models of this type have been obtained within the context of two-equation turbulence modeling by more formal expansion techniques incorporating, for example, the Direct Interaction Approximation (DIA) and the Renormalization Group (RNG) (see Yoshizawa 1984, Speziale 1987, Rubinstein and Barton 1990, and Yakhot et al. 1992). These models, which are characterized by an explicit relationship between the Reynolds stress tensor and the mean velocity gradients (and possibly their time derivatives) have come to be referred to as "anisotropic eddy viscosity models."

During the 1970's, second-order closure models became popular in which closure was achieved based on the Reynolds stress transport equation where history and nonlocal effects are accounted for. By means of an equilibrium hypothesis, in which the Reynolds stress convection and transport terms were neglected, algebraic stress models were obtained from second-order closures (see Rodi 1976). In these models, the Reynolds stresses were related 
implicitly to the mean velocity gradients. This provided the first formal means, based on a higher-order closure, to justify the extension of the Boussinesq hypothesis to incorporate nonlinearities in the mean velocity gradients. However, this type of algebraic stress model is cumbersome to implement in complex flows since the stress-strain relation is not explicit; numerical stiffness problems can result from the need for successive matrix inversions at each iteration.

In an interesting paper that has to a large extent gone unnoticed, Pope (1975) developed a nonlinear Reynolds stress model by invoking the same equilibrium hypothesis as Rodi (1976). However, Pope (1975) actually presented a methodology for obtaining an explicit relation for the Reynolds stress tensor from the implicit algebraic equations that Rodi (1976) obtained from the Launder, Reece and Rodi (1975) model. This methodology, which leads to explicit algebraic stress models, is based on the use of integrity bases from linear algebra. Due to the complexity of the algebra, Pope (1975) was only able to obtain a solution for two-dimensional turbulent flows which he accomplished using the Launder, Reece and Rodi model.

The main purpose of the present paper is to extend the results of Pope (1975) to threedimensional turbulent flows in non-inertial frames starting from a more general hierarchy of second-order closure models - a task that is now feasible computationally via symbolic manipulation. The relationship between these new explicit algebraic stress models and anisotropic eddy viscosity models, as well as the older nonlinear Reynolds stress models, will be established in a systematic fashion. There appears to be considerable confusion in the turbulence literature concerning the relationship between these various types of nonlinear Reynolds stress models that needs to be clarified. It will be shown that the new explicit algebraic stress models represent the equilibrium Reynolds stress anisotropies predicted by secondorder closures in homogeneous turbulent flows. Consequently, beyond their potential use in practical turbulence calculations, the explicit algebraic stress models to be derived herein can be used to explore the predictive capabilities of a hierarchy of second-order closures in homogeneous turbulence. These issues will be discussed in more detail in the sections to follow.

\section{THEORETICAL BACKGROUND}

The incompressible turbulent flow of a viscous fluid will be considered where the velocity $\mathrm{v}$ and kinematic pressure $P$ are decomposed into ensemble mean and fluctuating parts as follows:

$$
\mathbf{v}=\overline{\mathbf{v}}+\mathrm{u}, \quad P=\bar{P}+p
$$


The Reynolds stress tensor $\tau_{i j} \equiv \overline{u_{i} u_{j}}$ is a solution of the transport equation (c.f. Hinze 1975):

$$
\frac{D \tau_{i j}}{D t}=-\tau_{i k} \frac{\partial \bar{v}_{j}}{\partial x_{k}}-\tau_{j k} \frac{\partial \bar{v}_{i}}{\partial x_{k}}+\Phi_{i j}-\varepsilon_{i j}-2 \Omega_{m}\left(e_{m k j} \tau_{i k}+e_{m k i} \tau_{j k}\right)+\mathcal{D}_{i j}^{T}+\nu \nabla^{2} \tau_{i j}
$$

which is valid in an arbitrary non-inertial reference frame that can undergo a rotation with angular velocity $\Omega_{i}$ relative to an inertial framing. In (2), $D / D t \equiv \partial / \partial t+\overline{\mathbf{v}} \cdot \boldsymbol{\nabla}$ is the convective derivative, $e_{i j k}$ is the permutation tensor, $\nu$ is the kinematic viscosity, and

$$
\begin{gathered}
\Phi_{i j}=\overline{p\left(\frac{\partial u_{i}}{\partial x_{j}}+\frac{\partial u_{j}}{\partial x_{i}}\right)} \\
\varepsilon_{i j}=\overline{\nu\left(\frac{\partial u_{i}}{\partial x_{k}} \frac{\partial u_{j}}{\partial x_{k}}\right)} \\
\mathcal{D}_{i j}^{T}=-\frac{\partial}{\partial x_{k}}\left(\overline{u_{i} u_{j} u_{k}}+\overline{p u_{i}} \delta_{j k}+\overline{p u_{j}} \delta_{i k}\right)
\end{gathered}
$$

are, respectively, the pressure-strain correlation, the dissipation rate tensor, and the turbulent transport term. Turbulence models based on the Reynolds stress transport equation (2) are referred to as "second-order closures" since (2) is obtained by taking a second moment of the fluctuating Navier-Stokes equation.

Homogeneous turbulent flows in equilibrium, as well as regions of inhomogeneous turbulent flows where there is a production-equals-dissipation equilibrium, satisfy the constraints:

$$
\begin{gathered}
\frac{D b_{i j}}{D t}=0 \\
\mathcal{D}_{i j}^{T}+\nu \nabla^{2} \tau_{i j}=0
\end{gathered}
$$

where

$$
b_{i j}=\frac{\tau_{i j}-\frac{2}{3} K \delta_{i j}}{2 K}
$$

is the anisotropy tensor ( $K \equiv \frac{1}{2} \overline{u_{i} u_{i}}$ is the turbulent kinetic energy). In physical terms, this is an equilibrium for which convective and transport effects can be neglected; it is the basic equilibrium hypothesis used in the derivation of algebraic stress models. Although it constitutes an idealization, it is comforting to know that this equilibrium hypothesis is achievable in interesting physical limits that include homogeneous shear flow and the logarithmic region of an equilibrium turbulent boundary layer.

It follows from (6) that

$$
\frac{D \tau_{i j}}{D t}=\frac{\tau_{i j}}{K} \frac{D K}{D t}
$$


and, hence, by making use of the contraction of (2) and (7), we obtain

$$
\frac{D \tau_{i j}}{D t}=(\mathcal{P}-\varepsilon) \frac{\tau_{i j}}{K}
$$

where $\mathcal{P} \equiv-\tau_{i j} \partial \bar{v}_{i} / \partial x_{j}$ is the turbulence production and $\varepsilon \equiv \frac{1}{2} \varepsilon_{i i}$ is the scalar turbulent dissipation rate. The substitution of (7) and (10) into (2) yields the following equilibrium form of the Reynolds stress transport equation:

$$
(\mathcal{P}-\varepsilon) \frac{\tau_{i j}}{K}=-\tau_{i k} \frac{\partial \bar{v}_{j}}{\partial x_{k}}-\tau_{j k} \frac{\partial \bar{v}_{i}}{\partial x_{k}}+\Phi_{i j}-\varepsilon_{i j}-2 \Omega_{m}\left(e_{m k j} \tau_{i k}+e_{m k i} \tau_{j k}\right)
$$

The dissipation rate tensor can be split into isotropic and deviatoric parts as follows

$$
\varepsilon_{i j}=\frac{2}{3} \varepsilon \delta_{i j}+{ }_{D} \varepsilon_{i j}
$$

By making use of (8) and (12), we can rearrange (11) into the alternative form

$$
\begin{aligned}
(\mathcal{P}-\varepsilon) b_{i j}= & -\frac{2}{3} K \bar{S}_{i j}-K\left(b_{i k} \bar{S}_{j k}+b_{j k} \bar{S}_{i k}\right. \\
& \left.-\frac{2}{3} b_{m n} \bar{S}_{m n} \delta_{i j}\right)-K\left[b_{i k}\left(\bar{\omega}_{j k}+2 e_{m k j} \Omega_{m}\right)\right. \\
& \left.+b_{j k}\left(\bar{\omega}_{i k}+2 e_{m k i} \Omega_{m}\right)\right]+\frac{1}{2} \Pi_{i j}
\end{aligned}
$$

where

$$
\begin{gathered}
\bar{S}_{i j}=\frac{1}{2}\left(\frac{\partial \bar{v}_{i}}{\partial x_{j}}+\frac{\partial \bar{v}_{j}}{\partial x_{i}}\right), \quad \bar{\omega}_{i j}=\frac{1}{2}\left(\frac{\partial \bar{v}_{i}}{\partial x_{j}}-\frac{\partial \bar{v}_{j}}{\partial x_{i}}\right) \\
\Pi_{i j}=\Phi_{i j}-{ }_{D} \varepsilon_{i j} .
\end{gathered}
$$

In all of the commonly used second-order closure models, $\Pi_{i j}$ is modeled in the general form (see Lumley 1978, Reynolds 1987 and Speziale 1991):

$$
\Pi_{i j}=\varepsilon \mathcal{A}_{i j}(\mathbf{b})+K \mathcal{M}_{i j k l}(\mathbf{b}) \frac{\partial \bar{v}_{k}}{\partial x_{l}} .
$$

For non-inertial frames, $\partial \bar{v}_{k} / \partial x_{l}$ is replaced with $\partial \bar{v}_{k} / \partial x_{l}+e_{m l k} \Omega_{m}$ in (16). The substitution of (16) (with its non-inertial correction) into (13) yields a closed system of algebraic equations for the determination of the Reynolds stress anisotropy in terms of the mean velocity gradients. This constitutes the general form of algebraic stress models; the algebraic stress model of Rodi (1976) is obtained when the Launder, Reece and Rodi model for $\Pi_{i j}$ is introduced into (13) and non-inertial effects are neglected. It is clear that these algebraic stress models are implicit in nature since the Reynolds stress tensor appears on both sides of the equation. In the next section we will examine how explicit relations can be obtained from (13). 


\section{EXPLICIT ALGEBRAIC STRESS MODELS}

When models for $\Pi_{i j}$ that are tensorially linear in the anisotropy tensor $b_{i j}$ are considered, it is possible to obtain an explicit expression for $b_{i j}$ in terms of the mean velocity gradients. This can be accomplished by techniques from linear algebra that make use of integrity bases (namely, polynomial representations for isotropic tensor functions). Pope (1975) was the first to show this by basing his analysis on the Launder, Reece and Rodi model which is the most commonly used model for $\Pi_{i j}$ that is a linear function of $b_{i j}$. We will extend the analysis of Pope (1975) to a more general hierarchy of linear models for $\Pi_{i j}$ where non-inertial effects are accounted for. Furthermore, our analysis will be conducted for three-dimensional turbulent flows. Pope (1975) restricted his analysis to two-dimensional turbulent flows because of the severe complexity of the algebra - an obstacle that we will overcome by using Mathematica ${ }^{\mathrm{TM}}$ (Wolfram 1988).

It can be shown that the most general form of (16) that is tensorially linear in the anisotropy tensor $b_{i j}$ is given by (c.f. Reynolds 1987 and Speziale 1991):

$$
\begin{aligned}
\Pi_{i j}= & -C_{1} \varepsilon b_{i j}+C_{2} K \bar{S}_{i j}+C_{3} K\left(b_{i k} \bar{S}_{j k}+b_{j k} \bar{S}_{i k}-\frac{2}{3} b_{m n} \bar{S}_{m n} \delta_{i j}\right) \\
& +C_{4} K\left(b_{i k} \bar{W}_{j k}+b_{j k} \bar{W}_{i k}\right)
\end{aligned}
$$

where

$$
\bar{W}_{i j}=\bar{\omega}_{i j}+\varepsilon_{m j i} \Omega_{m}
$$

is the absolute vorticity tensor (namely, the vorticity tensor $\bar{\omega}_{i j}$ relative to an inertial frame). The coefficients $C_{1}-C_{4}$ can be functions of the invariants of $b_{i j}$ and can depend on $\mathcal{P} / \varepsilon$ (of course, (16) requires that, at most, $C_{1}$ is a linear function of $\mathcal{P} / \varepsilon$ ). The model of Launder, Reece and Rodi (1975), the model of Gibson and Launder (1978) and the linearized model of Speziale, Sarkar and Gatski (1991) - where the quadratic part of the slow pressure-strain correlation is neglected - are special cases of (17). For these models, we have the following coefficients:

Launder, Reece and Rodi Model

$$
C_{1}=3.0, C_{2}=0.8, C_{3}=1.75, C_{4}=1.31
$$

Gibson and Launder Model

$$
C_{1}=3.6, C_{2}=0.8, C_{3}=1.2, C_{4}=1.2
$$


Speziale, Sarkar and Gatski Model

$$
C_{1}=3.4+1.8 \mathcal{P} / \varepsilon, C_{2}=0.8-1.3 I_{b}^{1 / 2}, C_{3}=1.25, C_{4}=0.40
$$

where $I I_{b}=b_{i j} b_{i j}$. It should be noted that for sufficiently small anisotropies, more complicated models can be approximated by the form (17).

The direct substitution of (17) into (13) yields the equation:

$$
\begin{aligned}
b_{i j}= & \frac{1}{2} g \tau\left\{\left(C_{2}-\frac{4}{3}\right) \bar{S}_{i j}+\left(C_{3}-2\right)\left(b_{i k} \bar{S}_{j k}+b_{j k} \bar{S}_{i k}-\frac{2}{3} b_{m n} \bar{S}_{m n} \delta_{i j}\right)\right. \\
& \left.+\left(C_{4}-2\right)\left[b_{i k}\left(\bar{\omega}_{j k}+\frac{C_{4}-4}{C_{4}-2} e_{m k j} \Omega_{m}\right)+b_{j k}\left(\bar{\omega}_{i k}+\frac{C_{4}-4}{C_{4}-2} e_{m k i} \Omega_{m}\right)\right]\right\}
\end{aligned}
$$

where

$$
\begin{gathered}
g=\left(\frac{C_{1}}{2}+\frac{\mathcal{P}}{\varepsilon}-1\right)^{-1} \\
\tau=\frac{K}{\varepsilon} .
\end{gathered}
$$

If we introduce the dimensionless, rescaled variables:

$$
\begin{gathered}
S_{i j}^{*}=\frac{1}{2} g \tau\left(2-C_{3}\right) \bar{S}_{i j} \\
W_{i j}^{*}=\frac{1}{2} g \tau\left(2-C_{4}\right)\left[\bar{\omega}_{i j}+\left(\frac{C_{4}-4}{C_{4}-2}\right) e_{m j i} \Omega_{m}\right] \\
b_{i j}^{*}=\left(\frac{C_{3}-2}{C_{2}-\frac{4}{3}}\right) b_{i j}
\end{gathered}
$$

then (19) reduces to the simpler form

$$
b_{i j}^{*}=-S_{i j}^{*}-\left(b_{i k}^{*} S_{j k}^{*}+b_{j k}^{*} S_{i k}^{*}-\frac{2}{3} b_{k l}^{*} S_{k l}^{*} \delta_{i j}\right)+b_{i k}^{*} W_{k j}^{*}+b_{j k}^{*} W_{k i}^{*} .
$$

In matrix form, (28) can be written as

$$
\mathbf{b}^{*}=-\mathbf{S}^{*}-\left(\mathbf{b}^{*} \mathbf{S}^{*}+\mathbf{S}^{*} \mathbf{b}^{*}-\frac{2}{3}\left\{\mathbf{b}^{*} \mathbf{S}^{*}\right\} \mathbf{I}\right)+\mathbf{b}^{*} \mathbf{W}^{*}-\mathbf{W}^{*} \mathbf{b}^{*}
$$

where $\{\cdot\}$ denotes the trace and $\mathbf{I}$ denotes the unit tensor. This is a linear algebraic equation for the determination of $\mathbf{b}^{*}$ in terms of $\mathbf{S}^{*}$ and $\mathbf{W}^{*}$; the solution to (29) is of the general form

$$
\mathbf{b}^{*}=\mathbf{f}\left(\mathbf{S}^{*}, \mathbf{W}^{*}\right) .
$$

Form invariance under an orthogonal coordinate transformation (with rotation tensor $\mathbf{Q}$ ) requires that

$$
\mathbf{Q f}\left(\mathbf{S}^{*}, \mathbf{W}^{*}\right) \mathbf{Q}^{T}=\mathbf{f}\left(\mathbf{Q} \mathbf{S}^{*} \mathbf{Q}^{T}, \mathbf{Q} \mathbf{W}^{*} \mathbf{Q}^{T}\right)
$$


i.e., that $f$ be an isotropic tensor function of its arguments. It can be shown that the satisfaction of (31) requires that

$$
\mathbf{b}^{*}=\sum_{\lambda} G^{(\lambda)} \mathbf{T}^{(\lambda)}
$$

where $\mathbf{T}^{(\lambda)}$ is the integrity basis for functions of a symmetric and antisymmetric tensor and $G^{(\lambda)}$ are scalar functions of the irreducible invariants of $\mathbf{S}^{*}$ and $\mathbf{W}^{*}$. For the case under consideration, the integrity basis is given by (see Spencer 1971 and Pope 1975):

$$
\begin{array}{ll}
\mathbf{T}^{(1)}=\mathbf{S}^{*} & \mathbf{T}^{(6)}=\mathbf{W}^{* 2} \mathbf{S}^{*}+\mathbf{S}^{*} \mathbf{W}^{* 2}-\frac{2}{3}\left\{\mathbf{S}^{*} \mathbf{W}^{* 2}\right\} \mathbf{I} \\
\mathbf{T}^{(2)}=\mathbf{S}^{*} \mathbf{W}^{*}-\mathbf{W}^{*} \mathbf{S}^{*} & \mathbf{T}^{(7)}=\mathbf{W}^{*} \mathbf{S}^{*} \mathbf{W}^{* 2}-\mathbf{W}^{* 2} \mathbf{S}^{*} \mathbf{W}^{*} \\
\mathbf{T}^{(3)}=\mathbf{S}^{* 2}-\frac{1}{3}\left\{\mathbf{S}^{* 2}\right\} \mathbf{I} & \mathbf{T}^{(8)}=\mathbf{S}^{*} \mathbf{W}^{*} \mathbf{S}^{* 2}-\mathbf{S}^{* 2} \mathbf{W}^{*} \mathbf{S}^{*} \\
\mathbf{T}^{(4)}=\mathbf{W}^{* 2}-\frac{1}{3}\left\{\mathbf{W}^{* 2}\right\} \mathbf{I} & \mathbf{T}^{(9)}=\mathbf{W}^{* 2} \mathbf{S}^{* 2}+\mathbf{S}^{* 2} \mathbf{W}^{* 2}-\frac{2}{3}\left\{\mathbf{S}^{* 2} \mathbf{W}^{* 2}\right\} \mathbf{I} \\
\mathbf{T}^{(5)}=\mathbf{W}^{*} \mathbf{S}^{* 2}-\mathbf{S}^{* 2} \mathbf{W}^{*} & \mathbf{T}^{(10)}=\mathbf{W}^{*} \mathbf{S}^{* 2} \mathbf{W}^{* 2}-\mathbf{W}^{* 2} \mathbf{S}^{* 2} \mathbf{W}^{*}
\end{array}
$$

The irreducible invariants of $\mathbf{S}^{*}$ and $\mathbf{W}^{*}$ are:

$$
\begin{gathered}
\eta_{1}=\left\{\mathbf{S}^{* 2}\right\} \\
\eta_{2}=\left\{\mathbf{W}^{* 2}\right\} \\
\eta_{3}=\left\{\mathbf{S}^{* 3}\right\} \\
\eta_{4}=\left\{\mathbf{S}^{*} \mathbf{W}^{* 2}\right\} \\
\eta_{5}=\left\{\mathbf{S}^{* 2} \mathbf{W}^{* 2}\right\} .
\end{gathered}
$$

Hence, we have

$$
G^{(\lambda)}=G^{(\lambda)}\left(\eta_{1}, \eta_{2}, \eta_{3}, \eta_{4}, \eta_{5}\right), \quad \lambda=1,2, \ldots 10 .
$$

Pope (1975) showed that for two-dimensional flows, only $\mathbf{T}^{(1)}, \mathbf{T}^{(2)}$ and $\mathbf{T}^{(3)}$, as well as $\eta_{1}$ and $\eta_{2}$, are independent. Consequently, the calculations become much simpler. Here, we will obtain the three-dimensional solution using the same methodology that Pope used.

The direct substitution of (32) into (28) yields

$$
\begin{aligned}
\sum_{\lambda} G^{(\lambda)} \mathbf{T}^{(\lambda)}= & -\sum_{\lambda} \delta_{1 \lambda} \mathbf{T}^{(\lambda)}-\sum_{\lambda} G^{(\lambda)}\left[\mathbf{T}^{(\lambda)} \mathbf{S}^{*}+\mathbf{S}^{*} \mathbf{T}^{(\lambda)}-\frac{2}{3}\left\{\mathbf{T}^{(\lambda)} \mathbf{S}^{*}\right\} \mathbf{I}\right. \\
& \left.-\mathbf{T}^{(\lambda)} \mathbf{W}^{*}+\mathbf{W}^{*} \mathbf{T}^{(\lambda)}\right]
\end{aligned}
$$

where we have made use of the fact that $\mathbf{T}^{(1)}=\mathbf{S}^{*}$. Since $\mathbf{T}^{(\lambda)}$ is an integrity basis we know that

$$
\mathbf{T}^{(\lambda)} \mathbf{S}^{*}+\mathbf{S}^{*} \mathbf{T}^{(\lambda)}-\frac{2}{3}\left\{\mathbf{T}^{(\lambda)} \mathbf{S}^{*}\right\} \mathbf{I}=\sum_{\gamma} H_{\lambda \gamma} \mathbf{T}^{(\gamma)}
$$




$$
\mathbf{T}^{(\lambda)} \mathbf{W}^{*}-\mathbf{W}^{*} \mathbf{T}^{(\lambda)}=\sum_{\gamma} J_{\lambda \gamma} \mathbf{T}^{(\gamma)}
$$

(i.e., any polynomial in $\mathbf{S}^{*}$ and $\mathbf{W}^{*}$ can be expanded in the integrity bases; see Spencer 1971 and Pope 1975). The $10 \times 10$ matrices $H_{\lambda \gamma}$ and $J_{\lambda \gamma}$ are obtained by successive applications of the Cayley-Hamilton Theorem (the elements of these matrices are given in Appendix A). By substituting (41) and (42) into (40), we obtain an equation from which $G^{(\lambda)}$ can be determined:

$$
G^{(\lambda)}=-\delta_{1 \lambda}-\sum_{\gamma} G^{(\gamma)} H_{\gamma \lambda}+\sum_{\gamma} G^{(\gamma)} J_{\gamma \lambda}
$$

This is a $10 \times 10$ linear system of equations for the determination of the $G^{(\lambda)}$ which can be written in the matrix form

$$
\mathbf{A} \mathbf{G}=\mathbf{B}
$$

where the components of $\mathbf{A}, \mathbf{G}$ and $\mathbf{B}$ are given by

$$
\begin{gathered}
A_{\gamma \lambda}=-\delta_{\lambda \gamma}-H_{\lambda \gamma}+J_{\lambda \gamma} \\
G_{\lambda}=G^{(\lambda)} \\
B_{\lambda}=\delta_{1 \lambda}
\end{gathered}
$$

(see Appendix A). The solution to (44) is given by

$$
G^{(\lambda)}=A_{\lambda 1}^{-1}
$$

In order to obtain a closed form expression for $G^{(\lambda)}$, it is necessary to analytically invert the matrix $\mathbf{A}$. The manual inversion of matrix $\mathbf{A}$ is not feasible in light of its highly complex structure; however, such a mathematical computation can now be done symbolically by Mathematica $^{\mathrm{TM}}$ (Wolfram 1988). The resulting inverse matrix $\mathbf{A}^{-1}$ is quite complex, but as noted in (48), only the first column of its elements are needed. The resulting solution for $G^{(\lambda)}$ is:

$$
\begin{array}{ll}
G^{(1)}=-\frac{1}{2}\left(6-3 \eta_{1}-21 \eta_{2}-2 \eta_{3}+30 \eta_{4}\right) / D & G^{(6)}=9 / D \\
G^{(2)}=-\left(3+3 \eta_{1}-6 \eta_{2}+2 \eta_{3}+6 \eta_{4}\right) / D & G^{(7)}=9 / D \\
G^{(3)}=\left(6-3 \eta_{1}-12 \eta_{2}-2 \eta_{3}-6 \eta_{4}\right) / D & G^{(8)}=9 / D \\
G^{(4)}=-3\left(3 \eta_{1}+2 \eta_{3}+6 \eta_{4}\right) / D & G^{(9)}=18 / D \\
G^{(5)}=9 / D & G^{(10)}=0
\end{array}
$$

where the denominator $D$ is given by

$$
\begin{aligned}
D= & 3-\frac{7}{2} \eta_{1}+\eta_{1}^{2}-\frac{15}{2} \eta_{2}-8 \eta_{1} \eta_{2}+3 \eta_{2}^{2}-\eta_{3}+\frac{2}{3} \eta_{1} \eta_{3} \\
& -2 \eta_{2} \eta_{3}+21 \eta_{4}+24 \eta_{5}+2 \eta_{1} \eta_{4}-6 \eta_{2} \eta_{4}
\end{aligned}
$$


Unfortunately, this three-dimensional form is significantly more complex than the twodimensional form derived by Pope (1975). Nevertheless, the result presented in (49) can be shown to reduce to Pope's form in the two-dimensional limit where the invariants $\eta_{3}$ and $\eta_{4}$ are zero and $\eta_{5}$ becomes equal to $\frac{1}{2} \eta_{1} \eta_{2}$. Using this reduction and the redundancies in the integrity basis $\left(\mathbf{T}^{(4)} \cdots \mathbf{T}^{(10)}\right.$ are linear combinations of $\mathbf{T}^{(1)}, \mathbf{T}^{(2)}$ and $\mathbf{T}^{(3)}$; see Pope 1975), the resulting expression for $G^{(\lambda)}$ in the two-dimensional limit becomes

$$
\begin{aligned}
& G^{(1)}=-\frac{3}{3-2 \eta_{1}-6 \eta_{2}} \\
& G^{(2)}=-\frac{3}{3-2 \eta_{1}-6 \eta_{2}} \\
& G^{(3)}=\frac{6}{3-2 \eta_{1}-6 \eta_{2}} .
\end{aligned}
$$

The anisotropy tensor $\mathbf{b}^{*}$ is then given by

$$
\mathbf{b}^{*}=-\frac{3}{3-2 \eta_{1}-6 \eta_{2}}\left[\mathbf{S}^{*}+\left(\mathbf{S}^{*} \mathbf{W}^{*}-\mathbf{W}^{*} \mathbf{S}^{*}\right)-2\left(\mathbf{S}^{* 2}-\frac{1}{3}\left\{\mathbf{S}^{* 2}\right\} \mathbf{I}\right)\right],
$$

which can be shown to be identical to the expression in Pope (1975) after using (25)-(27) along with the constants (19) of the Launder, Reece and Rodi model. It should be noted that Pope used an alternative form for the third basis in (52) - constructed from the two and three dimensional Kronecker delta - that is directly proportional to our $\mathrm{T}^{(3)}$.

It is now clear that the model derived by Pope (1975) (which is a special case of (52)) actually constitutes the explicit solution of the algebraic stress model of Rodi for $\mathbf{b}$ in terms of $\overline{\mathbf{S}}$ and $\bar{\omega}$. Whereas Pope's analysis is only valid for two-dimensional flows in an inertial frame, we have now succeeded - by means of the representation theorem embodied in (32) - to extend his result to three-dimensional flows in non-inertial frames.

Some comments are in order concerning how the three-dimensional solution obtained herein compares with the result derived recently by Taulbee (1992). The three-dimensional result of Taulbee only contains the bases $\mathbf{T}^{(1)}, \mathbf{T}^{(2)}, \mathbf{T}^{(4)}, \mathbf{T}^{(6)}, \mathbf{T}^{(7)}$ where the coefficients $G^{(\lambda)}$, for the most part, only depend on the invariant $\eta_{2}$. This simplification results from the neglect of the second strain-dependent term in the brackets on the r.h.s. of (22) (namely, it is obtained by setting $C_{3}=2$ ). We feel that this is a questionable approximation. Our research on homogeneous shear flow indicates that the coefficient $C_{3}-2$ is nonzero and must be nearly half as large as the coefficient $C_{4}-2$ in order to obtain a good description of shear flows. Furthermore, by neglecting this term in (22), the coefficients of the solution become virtually independent of the strain rate $\bar{S}_{i j}$ and the model collapses to the standard $K-\varepsilon$ model in the absence of rotational strains. This stands in serious contradiction of the exact two-dimensional result (52) as well as our new three-dimensional result. 


\section{COMPARISON WITH OTHER NONLINEAR REYNOLDS STRESS MODELS}

The explicit algebraic stress model that we derived in Eqs. (32) and (49) formally constitutes the equilibrium anisotropy tensor predicted by a hierarchy of second-order closure models for homogeneous turbulence. These second-order closures are typically solved in conjunction with a modeled transport for the turbulent dissipation rate that is of the general form

$$
\frac{D \varepsilon}{D t}=C_{\varepsilon 1} \frac{\varepsilon}{K} \mathcal{P}-C_{\varepsilon 2} \frac{\varepsilon^{2}}{K}+C_{\varepsilon} \frac{\partial}{\partial x_{i}}\left(\frac{K}{\varepsilon} \tau_{i j} \frac{\partial \varepsilon}{\partial x_{j}}\right)
$$

where $C_{\varepsilon 1}, C_{\varepsilon 2}$ and $C_{\varepsilon}$ are either constants, or functions of the invariants of $\mathbf{b}$, that vary from one model to the next. For homogeneous turbulence, the transport term on the r.h.s. of (53) vanishes and the turbulent kinetic energy is a solution of the transport equation

$$
\frac{D K}{D t}=\mathcal{P}-\varepsilon
$$

From (53)-(54), it follows that $\mathcal{P} / \varepsilon$ achieves the following equilibrium value (see Speziale 1991)

$$
\frac{\mathcal{P}}{\varepsilon}=\frac{C_{\varepsilon 2}-1}{C_{\varepsilon 1}-1}
$$

that will be used to determine $g$ in homogeneous turbulence. Hence, the explicit algebraic stress model derived herein can be utilized to systematically explore the equilibrium states predicted by a hierarchy of second-order closures in homogeneous turbulent flows.

As discussed earlier, the explicit algebraic stress model given by Eqs. (32) and (49) represents an exact solution to the general implicit algebraic stress model (22) that includes the model of Rodi (1976) as a special case. Consequently, by a direct analysis of this explicit model, new insights can be gained concerning the problems that the traditional algebraic stress models have had in the calculation of complex turbulent flows. The denominator of the coefficients $G^{(\lambda)}$ given in (49) contains a sum of positive and negative terms which has the potential to become zero rendering singular behavior. For equilibrium homogeneous turbulence this will not happen since $\eta_{1} \ldots \eta_{5}$ are constrained by the transport equations for $\tau_{i j}$ and $\varepsilon$ to yield a well behaved solution (see Speziale and Mac Giolla Mhuiris 1989). However, when an algebraic stress model is applied to complex non-equilibrium turbulent flows, where the underlying assumptions invoked in its derivation are no longer valid, singular behavior can result. This becomes a distinct possibility in turbulent flows that have localized strain rates that are large. When the traditional algebraic stress models of the form (22) are applied to turbulent flows with large strain rates where $D$ can approach zero, we would expect the model to be ill-behaved. This problem has been experienced in the practical calculation of 
complex turbulent flows where the Rodi algebraic stress model has at times failed to converge when solved iteratively (Demuren and Rodi 1984 and Demuren, private communication). Hence, it is clear that there is the need to regularize these explicit algebraic stress models. Such an approach has been used recently in the kinetic theory with considerable success (see Rosenau 1989).

The model derived herein can be regularized by a Padé approximation (this approach was recently used by Yakhot et al. 1992 in the derivation of their RNG based dissipation rate equation). The main idea behind this approach can be easily illustrated for the twodimensional model given in (52). For this case, the coefficient on the r.h.s. of (52) can be rewritten as

$$
\frac{3}{3-2 \eta_{1}-6 \eta_{2}}=\frac{3}{3-2 \eta^{2}+6 \zeta^{2}}
$$

where

$$
\eta=\left(S_{i j}^{*} S_{i j}^{*}\right)^{1 / 2}, \quad \zeta=\left(W_{i j}^{*} W_{i j}^{*}\right)^{1 / 2} .
$$

It is clear from (56) that, for sufficiently large strain rates $\eta$, singularities can occur; on the other hand, the rotational strains do not cause any problems. In an equilibrium homogeneous turbulent flow, $\eta$ is typically less than one. For example, in homogeneous shear flow (with shear rate $S$ ), the parameter $S K / \varepsilon$ achieves an equilibrium value of approximately 5 . For the Launder, Reece and Rodi model, this corresponds to $\eta \approx 0.2$ (see Speziale and Mac Giolla Mhuiris 1989). Hence, we want to replace (56) with a regular function that, for $\eta$ sufficiently less than one, is approximately the same. This can be accomplished by a Padé approximation. For example, to the first order we take

$$
\eta^{2} \approx 1-\frac{1}{1+\eta^{2}} .
$$

Substituting (58) into (56) then yields the expression

$$
\frac{3}{3-2 \eta_{1}-6 \eta_{2}} \approx \frac{3\left(1+\eta^{2}\right)}{3+\eta^{2}+6 \zeta^{2} \eta^{2}+6 \zeta^{2}}
$$

which is regular. Hence, we obtain a regularized model that is well-behaved for all strain rates and constitutes a good approximation to the original model within the equilibrium range that it formally applies. For practical applications, the regularized model

$$
\mathbf{b}^{*}=-\frac{3\left(1+\eta^{2}\right)}{3+\eta^{2}+6 \zeta^{2} \eta^{2}+6 \zeta^{2}}\left[\mathbf{S}^{*}+\left(\mathbf{S}^{*} \mathbf{W}^{*}-\mathbf{W}^{*} \mathbf{S}^{*}\right)-2\left(\mathbf{S}^{* 2}-\frac{1}{3}\left\{\mathbf{S}^{* 2}\right\} \mathbf{I}\right)\right]
$$

should be used instead of the form (52) first derived by Pope (1975) for the special case of the Launder, Reece and Rodi model. For the three-dimensional case, a more systematic Padé approximation to (50) must be made. This leads to rather cumbersome expressions that require further simplification and are currently under investigation. 
We will now show the relationship between the explicit algebraic stress model derived herein and existing anisotropic eddy viscosity models. It is clear that (32), with the coefficients (49), is of the general mathematical form

$$
b_{i j}^{*}=A_{i j k l}\left(\frac{\partial v_{k}}{\partial x_{l}}\right)^{*}
$$

where $\left(\partial v_{k} / \partial x_{l}\right)^{*} \equiv S_{k l}^{*}+W_{k l}^{*}$ and $A_{i j k l}$ is a fourth rank tensor that is a function of $\mathbf{S}^{*}$ and $\mathbf{W}^{*}$. This constitutes the general form of anisotropic eddy viscosity models; the standard eddy viscosity hypothesis of Boussinesq is recovered in the limit as $A_{i j k l}$ becomes an isotropic tensor.

Our explicit algebraic stress model for three-dimensional turbulent flows is tensorially quartic in the mean velocity gradients with coefficients that are ratios of polynomials in the invariants of $\overline{\mathbf{S}}$ and $\overline{\mathbf{W}}$. We will make use of the fact that

$$
\tau_{i j}=\frac{2}{3} K \delta_{i j}+2 \alpha_{1} K b_{i j}^{*}
$$

where $\alpha_{1}=\left(C_{2}-\frac{4}{3}\right) /\left(C_{3}-2\right)$. The normalized anisotropy tensor $\mathbf{b}^{*}$ can then be expanded in a Taylor series. To the first order in the mean velocity gradients, we have

$$
b_{i j}^{*}=-S_{i j}^{*}
$$

from the algebraic stress model (32) and (49). The direct substitution of (63) into (62), after making use of (25), yields

$$
\tau_{i j}=\frac{2}{3} K \delta_{i j}-2 C_{\mu}^{*} \frac{K^{2}}{\varepsilon} \bar{S}_{i j}
$$

where $C_{\mu}^{*}=\frac{1}{2} g\left(\frac{4}{3}-C_{2}\right)$. This is the eddy viscosity form of the standard $K-\varepsilon$ model (see Launder and Spalding 1974) with a coefficient $C_{\mu}^{*}$ that, for the second-order closures considered herein, is close to the traditional value of 0.09 . When quadratic terms are maintained, we have

$$
b_{i j}^{*}=-S_{i j}^{*}-\left(S_{i k}^{*} W_{k j}^{*}+S_{j k}^{*} W_{k i}^{*}\right)+2\left(S_{i k}^{*} S_{k j}^{*}-\frac{1}{3} S_{m n}^{*} S_{m n}^{*} \delta_{i j}\right)
$$

which, in an inertial frame, yields

$$
\tau_{i j}=\frac{2}{3} K \delta_{i j}-2 C_{\mu}^{*} \frac{K^{2}}{\varepsilon} \bar{S}_{i j}-\beta_{1} \frac{K^{3}}{\varepsilon^{2}}\left(\bar{S}_{i k} \bar{\omega}_{k j}+\bar{S}_{j k} \bar{\omega}_{k i}\right)+\beta_{2} \frac{K^{3}}{\varepsilon^{2}}\left(\bar{S}_{i k} \bar{S}_{k j}-\frac{1}{3} \bar{S}_{m n} \bar{S}_{m n} \delta_{i j}\right)
$$

where $\beta_{1}=\frac{1}{2} g^{2}\left(2-C_{4}\right)\left(\frac{4}{3}-C_{2}\right)$ and $\beta_{2}=g^{2}\left(2-C_{3}\right)\left(\frac{4}{3}-C_{2}\right)$. This is identical in form to the nonlinear $K-\varepsilon$ model of Speziale (1987) when convective effects are neglected. The nonlinear $K-\varepsilon$ model is given by

$$
\begin{aligned}
\tau_{i j}= & \frac{2}{3} K \delta_{i j}-2 C_{\mu} \frac{K^{2}}{\varepsilon} \bar{S}_{i j}-4 C_{D} C_{\mu}^{2} \frac{K^{3}}{\varepsilon^{2}}\left(\stackrel{\circ}{S}_{i j}-\frac{1}{3} \bar{S}_{m m} \delta_{i j}\right) \\
& -4 C_{D} C_{\mu}^{2} \frac{K^{3}}{\varepsilon^{2}}\left(\bar{S}_{i k} \bar{S}_{k j}-\frac{1}{3} \bar{S}_{m n} \bar{S}_{m n} \delta_{i j}\right)
\end{aligned}
$$


where $C_{D}$ and $C_{\mu}$ are constants and

$$
\stackrel{\circ}{S}_{i j}=\frac{D \bar{S}_{i j}}{D t}-\frac{\partial \bar{v}_{i}}{\partial x_{k}} \bar{S}_{k j}-\frac{\partial \bar{v}_{j}}{\partial x_{k}} \bar{S}_{k i}
$$

is the Oldroyd derivative of $\overline{\mathbf{S}}$. When $D \bar{S}_{i j} / D t$ is neglected, it is a simple matter to show that (67) is of the same form as (66).

Eq. (66) is also of the same form as the anisotropic eddy viscosity models of Yoshizawa (1984) and Rubinstein and Barton (1990) with one exception: it does not contain a term of the type

$$
\beta_{3} \frac{K^{3}}{\varepsilon^{2}}\left(\bar{\omega}_{i k} \bar{\omega}_{k j}-\frac{1}{3} \bar{\omega}_{m n} \bar{\omega}_{m n} \delta_{i j}\right)
$$

This kind of term was eliminated by Speziale (1987) because it yields an erroneous prediction for isotropic turbulence subjected to a solid body rotation. More precisely, a term of the form (69) predicts that an initially isotropic turbulence develops Reynolds stress anisotropies when subjected to a solid body rotation - a result that is in contradiction of physical and numerical experiments (see Wigeland and Nagib 1978 and Speziale, Mansour and Rogallo 1987). It is encouraging that this consistency constraint, concerning the vanishing of (69) to the second order in the mean velocity gradients, has now been obtained directly by a systematic analysis of the Reynolds stress transport equation.

It is interesting to note that the quadratic approximation (66) to the three-dimensional algebraic stress model is of the same tensorial form as the full nonlinear two-dimensional model (60). However, due to the strain-dependent coefficients in (60), more physics is accounted for. For example, Eq. (60) properly predicts that in a rapidly rotating frame - where $\Omega$, and hence $\zeta$, tends to infinity $-b_{i j}^{*}$ and $\mathcal{P}$ go to zero. This is the restabilization effect of turbulence in a rapidly rotating frame that the usual anisotropic eddy viscosity models are unable to predict (see Speziale, Gatski and Mac Giolla Mhuiris 1990). Hence, we feel that the two-dimensional algebraic stress model (60) should be tried in the future as an alternative to the more commonly used quadratic anisotropic eddy viscosity models. Some applications of $(60)$ will be considered in the next section.

\section{ILLUSTRATIVE EXAMPLES}

In order to demonstrate the efficacy of the regularized algebraic stress model (60) derived in this study, we will consider a few applications to non-trivial, two-dimensional turbulent flows involving shear and rotation. We will base our calculations on the Speziale, Sarkar and Gatski (1991) second-order closure (hereafter, referred to as the SSG model). The regularized 
algebraic stress model can be rewritten in the form

$$
\tau_{i j}=\frac{2}{3} K \delta_{i j}-\frac{6\left(1+\eta^{2}\right) \alpha_{1} K}{3+\eta^{2}+6 \zeta^{2} \eta^{2}+6 \zeta^{2}}\left[S_{i j}^{*}+\left(S_{i k}^{*} W_{k j}^{*}+S_{j k}^{*} W_{k i}^{*}\right)-2\left(S_{i k}^{*} S_{k j}^{*}-\frac{1}{3} S_{k l}^{*} S_{k l}^{*} \delta_{i j}\right)\right]
$$

where $\alpha_{1}=\left(C_{2}-\frac{4}{3}\right) /\left(C_{3}-2\right)$ and $\mathbf{S}^{*}, \mathbf{W}^{*}, \eta$ and $\zeta$ are as defined in (25), (26), and (57). Some remarks are needed concerning how $\mathcal{P} / \varepsilon$ and $I_{b}^{1 / 2}$ are evaluated in the model coefficients (21) for the SSG model. $\mathcal{P} / \varepsilon$ is calculated using (55) which is formally valid for equilibrium homogeneous turbulent flows; we take $I_{b} \approx 0.11$ which is the universal equilibrium value predicted by the SSG model for two-dimensional homogeneous turbulence (see Speziale, Sarkar and Gatski 1991). This yields the following choice of constants

$$
C_{1}=6.80, C_{2}=0.36, C_{3}=1.25, C_{4}=0.40, g=0.233
$$

for the explicit algebraic stress model corresponding to the SSG second-order closure.

The explicit algebraic stress model (70) is solved in conjunction with modeled transport equations for $K$ and $\varepsilon$. These equations are of the same general form as those used in the $K-\varepsilon$ model and are given by:

$$
\begin{gathered}
\frac{D K}{D t}=-\tau_{i j} \frac{\partial \bar{v}_{i}}{\partial x_{j}}-\varepsilon+\frac{\partial}{\partial x_{i}}\left(\frac{\nu_{T}}{\sigma_{K}} \frac{\partial K}{\partial x_{i}}\right) \\
\frac{D \varepsilon}{D t}=-C_{\varepsilon 1} \frac{\varepsilon}{K} \tau_{i j} \frac{\partial \bar{v}_{i}}{\partial x_{j}}-C_{\varepsilon 2} \frac{\varepsilon^{2}}{K}+\frac{\partial}{\partial x_{i}}\left(\frac{\nu_{T}}{\sigma_{\varepsilon}} \frac{\partial \varepsilon}{\partial x_{i}}\right)
\end{gathered}
$$

where $\nu_{T}=C_{\mu} K^{2} / \varepsilon, C_{\mu}=0.09, \sigma_{K}=1, \sigma_{\varepsilon}=1.3, C_{\varepsilon 1}=1.44$ and $C_{\varepsilon 2}=1.83$. Hence, the explicit algebraic stress model derived herein formally constitutes a two-equation turbulence model; the $K-\varepsilon$ model is recovered when (70) is linearized with respect to the mean velocity gradients. While this explicit algebraic stress model is a two-equation model, it incorporates much more physics than the $K-\varepsilon$ model since it is consistent with second-order closures in the limit of equilibrium homogeneous turbulent flows.

The first example that we will consider is the case of homogeneous shear flow in a rotating frame. An initially decaying isotropic turbulence is, at time $t=0$, subjected to a uniform shear rate $S$ in a reference frame rotating steadily with angular velocity $\Omega$. The corresponding mean velocity gradient tensor is given by

$$
\frac{\partial \bar{v}_{i}}{\partial x_{j}}=\left(\begin{array}{ccc}
0 & S & 0 \\
0 & 0 & 0 \\
0 & 0 & 0
\end{array}\right)
$$

and the angular velocity of the reference frame is given by $\Omega_{i}=(0,0, \Omega)$ (see Figure 1 ). In Table 1, the equilibrium values predicted by the new explicit algebraic stress model (ASM) 
for homogeneous shear flow are compared with the experimental data of Tavoularis and Corrsin (1981) as well as with the predictions of the SSG model and the standard $K-\varepsilon$ model. From these results it is clear that: (a) the new explicit ASM performs far better than the standard $K-\varepsilon$ model, and (b) the new explicit ASM yields results in close proximity of the full SSG second-order closure model. The small differences between the new ASM model and the full SSG model arises from two sources: the neglect of the quadratic return to isotropy term and the Padé approximation that was implemented to regularize the model. However, it is clear from these results that the new regularized ASM model constitutes an excellent approximation to the full SSG model for equilibrium flows.

Now, we will compare the predictions of these three models for the time-evolution of the turbulent kinetic energy $\left(K^{*} \equiv K / K_{0}, t^{*} \equiv S t\right)$ with the large-eddy simulations (LES) of Bardina, Ferziger and Reynolds (1983) for rotating homogeneous shear flow. In Figures 2(a)$2(\mathrm{c})$, the model predictions for three rotation rates $(\Omega / S=0, \Omega / S=0.5$ and $\Omega / S=-0.5)$ are compared with the LES which is for an initial condition of $\varepsilon_{0} / S K_{0}=0.296$. From these results it is clear that the new algebraic stress model does an excellent job in capturing the trends of the LES. It yields results that are far superior to the standard $K-\varepsilon$ model and are in close proximity to the SSG model. The main discernible difference between the new algebraic stress model and the SSG second-order closure is during the early transient where it responds more abruptly to the application of the shear since it does not account directly for relaxation effects. However, it is important to note how the new ASM is far superior to the standard $K-\varepsilon$ model in responding to changes in the rotation rate. This is illustrated in Figures 3(a)-3(c) where it can be seen that the new algebraic stress model is able to capture the effect of rotations on homogeneous shear flow. In contrast to these results, the standard $K-\varepsilon$ erroneously yields the same results for all rotation rates - a deficiency tied to the Boussinesq eddy viscosity hypothesis.

The last example that we will consider is the case of fully-developed turbulent channel flow subjected to a spanwise rotation with constant angular velocity $\Omega$ (see Figure 4). We will consider the experimental test case of Johnston, Halleen and Lezius (1972) for a Reynolds number $R e=11,500$ and a rotation number $R o=0.21$ (here, $R e=U_{0} H / \nu$ and $R o=$ $\Omega H / U_{0}$ where $U_{0}$ is the bulk mean velocity). In Figure 5(a) the prediction for the mean velocity profile obtained from the SSG second-order closure by Speziale, So and Younis (1992) is compared with the experimental data of Johnston, Halleen and Lezius (1972). The computations were done using law of the wall boundary conditions. In Figure 5(b), the same comparisons are made for the new explicit algebraic stress model (60) derived herein. It is clear from these results that the new ASM yields an asymmetric mean velocity profile that is very similar to that obtained from the full second-order closure. Due to the use of wall 
functions - as well as the neglect of roll instabilities (see Speziale, So and Younis 1992) - the specific quantitative comparisons are not that good. The important point, however, is that the new algebraic stress model yields an asymmetric mean velocity profile in close proximity to that obtained from a full second-order closure. On the other hand, simpler two-equation models like the standard $K-\varepsilon$ model erroneously predict that the mean velocity profile is unaffected by a system rotation and remains symmetric (see Speziale 1991). The improved predictions of the new explicit ASM in rotating shear flows arises from the incorporation of rotational strains through the terms $\mathbf{W}^{*}$ and $\zeta$.

\section{CONCLUSION}

Explicit algebraic stress models for two and three dimensional turbulent flows, in noninertial frames, have been obtained for a hierarchy of second-order closure models that are tensorially linear in the Reynolds stress anisotropy. These models were obtained using the standard local equilibrium hypothesis and, therefore, constitute the explicit solution to the traditional algebraic stress models generalized to include non-inertial effects and a range of pressure-strain models. They also formally represent the equilibrium states predicted by this hierarchy of second-order closures in homogeneous turbulent flows. A direct examination of these explicit models has shed new light on the limitations of the traditional algebraic stress models in applications to complex turbulent flows. For localized strain rates that are large, traditional algebraic stress models can become singular. These models need to be regularized - a task that can be achieved by means of a Padé approximation as demonstrated in this paper.

The results of this study have presented the first definitive evidence as to why the traditional algebraic stress models are ill-behaved, yielding numerical problems in many applications. These models should eventually be abandoned in favor of regularized versions of the explicit algebraic stress models derived herein. The full three-dimensional form of the explicit models is rather complicated and work is underway to simplify them by means of a rational approximation procedure. However, in the mean time, the regularized two-dimensional form $(60)$ can be used in practical applications. This simplified model is formally valid for two-dimensional equilibrium flows and collapses to the recently proposed anisotropic eddy viscosity models in the limit of small strain rates. However, while its general tensorial form is as simple as the commonly used anisotropic eddy viscosity models, it incorporates much more physics since the coefficients depend nonlinearly on both rotational and irrotational strains. A version of $(60)$ based on the SSG second-order closure was applied to rotating shear flows herein with encouraging results. These developments, when combined with some recent improvements in the modeling of the turbulent dissipation rate, can lead to a new 
generation of two-equation models that can serve as a useful companion to second-order closures in the calculation of complex turbulent flows.

\section{ACKNOWLEDGEMENTS}

The authors are indebted to Prof. G. F. Smith (Lehigh University) for some helpful comments concerning tensor representation theorems. The assistance of Prof. B. A. Younis (City University, London) with the rotating channel flow calculations is gratefully acknowledged. 


\section{APPENDIX A}

The elements of the matrices $H_{\gamma \lambda}$ and $J_{\gamma \lambda}$ are given in terms of $\eta_{i}$ as

$$
H_{\gamma \lambda}=\left[\begin{array}{cccccccccc}
0 & 0 & 2 & 0 & 0 & 0 & 0 & 0 & 0 & 0 \\
0 & 0 & 0 & 0 & -1 & 0 & 0 & 0 & 0 & 0 \\
\frac{\eta_{1}}{3} & 0 & 0 & 0 & 0 & 0 & 0 & 0 & 0 & 0 \\
\frac{-2 \eta_{2}}{3} & 0 & 0 & 0 & 0 & 1 & 0 & 0 & 0 & 0 \\
0 & \frac{-\eta_{1}}{2} & 0 & 0 & 0 & 0 & 0 & 1 & 0 & 0 \\
\frac{2 \eta_{1}}{3} & 0 & 2 \eta_{2} & \eta_{1} & 0 & 0 & 0 & 0 & -1 & 0 \\
0 & -\eta_{4} & 0 & 0 & \eta_{2} & 0 & 0 & 0 & 0 & -2 \\
0 & \frac{\eta_{3}}{3} & 0 & 0 & 0 & 0 & 0 & 0 & 0 & 0 \\
\frac{-\eta_{5}}{3} & 0 & \eta_{4} & \frac{\eta_{3}}{3} & 0 & \frac{\eta_{1}}{2} & 0 & 0 & 0 & 0 \\
0 & \frac{-\eta_{5}}{3}-\frac{\eta_{1} \eta_{2}}{6} & 0 & 0 & \frac{2 \eta_{4}}{3} & 0 & \frac{-\eta_{1}}{3} & \frac{\eta_{2}}{3} & 0 & 0
\end{array}\right]
$$

$$
J_{\gamma \lambda}=\left[\begin{array}{cccccccccc}
0 & 1 & 0 & 0 & 0 & 0 & 0 & 0 & 0 & 0 \\
-\eta_{2} & 0 & 0 & 0 & 0 & 3 & 0 & 0 & 0 & 0 \\
0 & 0 & 0 & 0 & -1 & 0 & 0 & 0 & 0 & 0 \\
0 & 0 & 0 & 0 & 0 & 0 & 0 & 0 & 0 & 0 \\
0 & 0 & \eta_{2} & 2 \eta_{1} & 0 & 0 & 0 & 0 & -3 & 0 \\
0 & \frac{\eta_{2}}{2} & 0 & 0 & 0 & 0 & -1 & 0 & 0 & 0 \\
\eta_{2}^{2} & 0 & 0 & -2 \eta_{4} & 0 & -2 \eta_{2} & 0 & 0 & 0 & 0 \\
2 \eta_{5}-\eta_{1} \eta_{2} & 0 & -2 \eta_{4} & 0 & 0 & \eta_{1} & 0 & 0 & 0 & 0 \\
0 & 0 & 0 & 0 & \frac{-\eta_{2}}{2} & 0 & 0 & 0 & 0 & -1 \\
0 & 0 & \eta_{2}^{2} & 2 \eta_{1} \eta_{2}-2 \eta_{5} & 0 & 0 & 0 & 0 & -2 \eta_{2} & 0
\end{array}\right]
$$


The elements of the matrix $\mathbf{A}$ are a linear combination of the elements of the matrices $H_{\gamma \lambda}$ and $J_{\gamma \lambda}$, as well as the identity matrix. The matrix structure is then given by

$$
\mathbf{A}=\left[\begin{array}{cccccccccc}
-1 & A_{12} & A_{13} & A_{14} & 0 & A_{16} & A_{17} & A_{18} & A_{19} & 0 \\
A_{21} & -1 & 0 & 0 & A_{25} & A_{26} & A_{27} & A_{28} & 0 & A_{210} \\
A_{31} & 0 & -1 & 0 & A_{35} & A_{36} & 0 & A_{38} & A_{39} & A_{310} \\
0 & 0 & 0 & -1 & A_{45} & A_{46} & A_{47} & 0 & A_{49} & A_{410} \\
0 & A_{52} & A_{53} & 0 & -1 & 0 & A_{57} & 0 & A_{59} & A_{510} \\
0 & 0 & 0 & A_{64} & 0 & -1 & A_{67} & A_{68} & A_{69} & 0 \\
0 & 0 & 0 & 0 & 0 & A_{76} & -1 & 0 & 0 & A_{710} \\
0 & 0 & 0 & 0 & A_{85} & 0 & 0 & -1 & 0 & A_{810} \\
0 & 0 & 0 & 0 & A_{95} & A_{96} & 0 & 0 & -1 & A_{910} \\
0 & 0 & 0 & 0 & 0 & 0 & A_{107} & 0 & A_{109} & -1
\end{array}\right]
$$

with the nonzero elements determined from

$$
A_{\gamma \lambda}=-\delta_{\lambda \gamma}-H_{\lambda \gamma}+J_{\lambda \gamma}
$$

The vector $\mathbf{B}$ is given by

$$
\mathbf{B}=\left[\begin{array}{l}
1 \\
0 \\
0 \\
0 \\
0 \\
0 \\
0 \\
0 \\
0 \\
0
\end{array}\right]
$$




\section{REFERENCES}

Bardina, J., Ferziger, J. H. and Reynolds, W. C. 1983. Improved turbulence models based on large-eddy simulation of homogeneous, incompressible turbulent flows. Stanford Univ. Technical Report No. TF-19.

Demuren, A. and Rodi, W. 1984. Calculation of turbulence-driven secondary motion in non-circular ducts. J. Fluid Mech. 140, 189.

Gibson, M. M. and Launder, B. E. 1978. Ground effects on pressure fluctuations in the atmospheric boundary layer. J. Fluid Mech. 86, 491.

Hinze, J. O. 1975. Turbulence. McGraw-Hill.

Johnston, J. P., Halleen, R. M. and Lezius, D. K. 1972. Effects of a spanwise rotation on the structure of two-dimensional fully-developed channel flow. J. Fluid Mech. 56, 533.

Launder, B. E. 1990. Phenomenological modeling: Present and future. Lecture Notes in Physics (ed. J. L. Lumley), Vol 357, p. 439, Springer-Verlag.

Launder, B. E. and Spalding, D. B. 1974. The numerical computation of turbulent flows. Computer Methods in Applied Mechanics and Engineering 3, 269.

Launder, B. E., Reece, G., and Rodi, W. 1975. Progress in the development of a Reynolds stress turbulence closure. J. Fluid Mech. 68, 537.

Lumley, J. L. 1970. Toward a turbulent constitutive equation. J. Fluid Mech. 41, 413.

Lumley, J. L. 1978. Computational modeling of turbulent flows. Adv. Appl. Mech. 18, 123.

Pope, S. B. 1975. A more general effective viscosity hypothesis. J. Fluid Mech. 72, 331.

Reynolds, W. C. 1987. Fundamentals of turbulence for turbulence modeling and simulation. Lecture Notes for Von Karman Institute, AGARD Lecture Series No. 86, North Atlantic Treaty Organization.

Rivlin, R. S. 1957. The relation between the flow of non-Newtonian fluids and turbulent Newtonian fluids. Q. Appl. Math. 15, 212.

Rodi, W. 1976. A new algebraic relation for calculating the Reynolds stresses. ZAMM 56, $\mathrm{T} 219$. 
Rosenau, P. 1989. Extending hydrodynamics via the regularization of the Chapman-Enskog expansion. Phys. Rev. A 40, 7193.

Rubinstein, R. and Barton, J. M. 1990. Nonlinear Reynolds stress models and the Renormalization group. Phys. Fluids A 2, 1472.

Saffman, P. G. 1977. Results of a two-equation model for turbulent flows and development of a relaxation stress model for application to straining and rotating flows. Proc. Project SQUID Workshop on Turbulence in Internal Flows (ed. S. Murthy), p. 191, Hemisphere Press.

Spencer, A. J. M. 1971. Theory of invariants. Continuum Physics (ed. A. C. Eringen), Vol. 1, p. 1, Academic Press.

Speziale, C. G. 1987. On nonlinear $K-\ell$ and $K-\varepsilon$ models of turbulence. J. Fluid Mcch. $\mathbf{1 7 8}, 459$.

Speziale, C. G. 1991. Analytical methods for the development of Reynolds stress closures in turbulence. Ann. Rev. Fluid Mech. 23, 107.

Speziale, C. G., Mansour, N. N., and Rogallo, R. S. 1987. The decay of isotropic turbulence in a rapidly rotating frame. Proceedings of the 1987 Summer Program of the Center for Turbulence Research (eds. P. Moin, W. C. Reynolds, and J. Kim), p. 205. Stanford University Press.

Speziale, C. G. and Mac Giolla Mhuiris, N. 1989. On the prediction of equilibrium states in homogeneous turbulence. J. Fluid Mech. 209, 591.

Speziale, C. G., Gatski, T. B., and Mac Giolla Mhuiris, N. 1990. A critical comparison of turbulence models for homogeneous shear flows in a rotating frame. Phys. Fluids A 2, 1678.

Speziale, C. G., Sarkar, S., and Gatski, T. B. 1991. Modeling the pressure-strain correlation of turbulence: an invariant dynamical systems approach. J. Fluid Mech. 227, 245.

Speziale, C. G., So, R. M. C. and Younis, B. A. 1992. On the prediction of turbulent secondary flows. ICASE Report No. 92-57, NASA Langley Research Center.

Taulbee, D. B. 1992. An improved algebraic Reynolds stress model and corresponding nonlinear stress model. Phys. Fluids A 4, 2555. 
Tavoularis, S. and Corrsin, S. 1981. Experiments in nearly homogeneous turbulent shear flow with a uniform mean temperature gradient. Part I. J. Fluid Mech. 104, 311.

Wigeland, R. A. and Nagib, H. M. 1978. Grid-generated turbulence with and without rotation about the streamwise direction. IIT Fluids and Heat Transfer Report R78-1.

Wolfram, S. 1988. Mathematica, Addison-Wesley Publishing Co.

Yakhot, V., Orszag, S. A., Thangam, S., Gatski, T. B., and Speziale, C. G. 1992. Development of turbulence models for shear flows by a double expansion technique. Phys. Fluids A 4, 1510.

Yoshizawa, A. 1984. Statistical analysis of the deviation of the Reynolds stress from its eddy viscosity representation. Phys. Fluids 27, 1377. 


\begin{tabular}{|c|c|c|c|c|}
\hline $\begin{array}{c}\text { Equilibrium } \\
\text { Values }\end{array}$ & $\begin{array}{c}\text { Standard } \\
K-\varepsilon \text { Model }\end{array}$ & $\begin{array}{c}\text { New ASM } \\
\text { Model }\end{array}$ & SSG Model & $\begin{array}{c}\text { Experimental } \\
\text { Data }\end{array}$ \\
\hline$b_{11}$ & 0 & 0.204 & 0.218 & 0.20 \\
\hline$b_{12}$ & -0.217 & -0.157 & -0.163 & -0.15 \\
\hline$b_{22}$ & 0 & -0.149 & -0.146 & -0.14 \\
\hline$b_{33}$ & 0 & -0.055 & -0.072 & -0.06 \\
\hline$S K / \varepsilon$ & 4.82 & 6.02 & 5.76 & 6.0 \\
\hline
\end{tabular}

Table 1. Comparison of the model predictions with the experimental equilibrium values in homogeneous shear flow measured by Tavoularis and Corrsin (1981). 


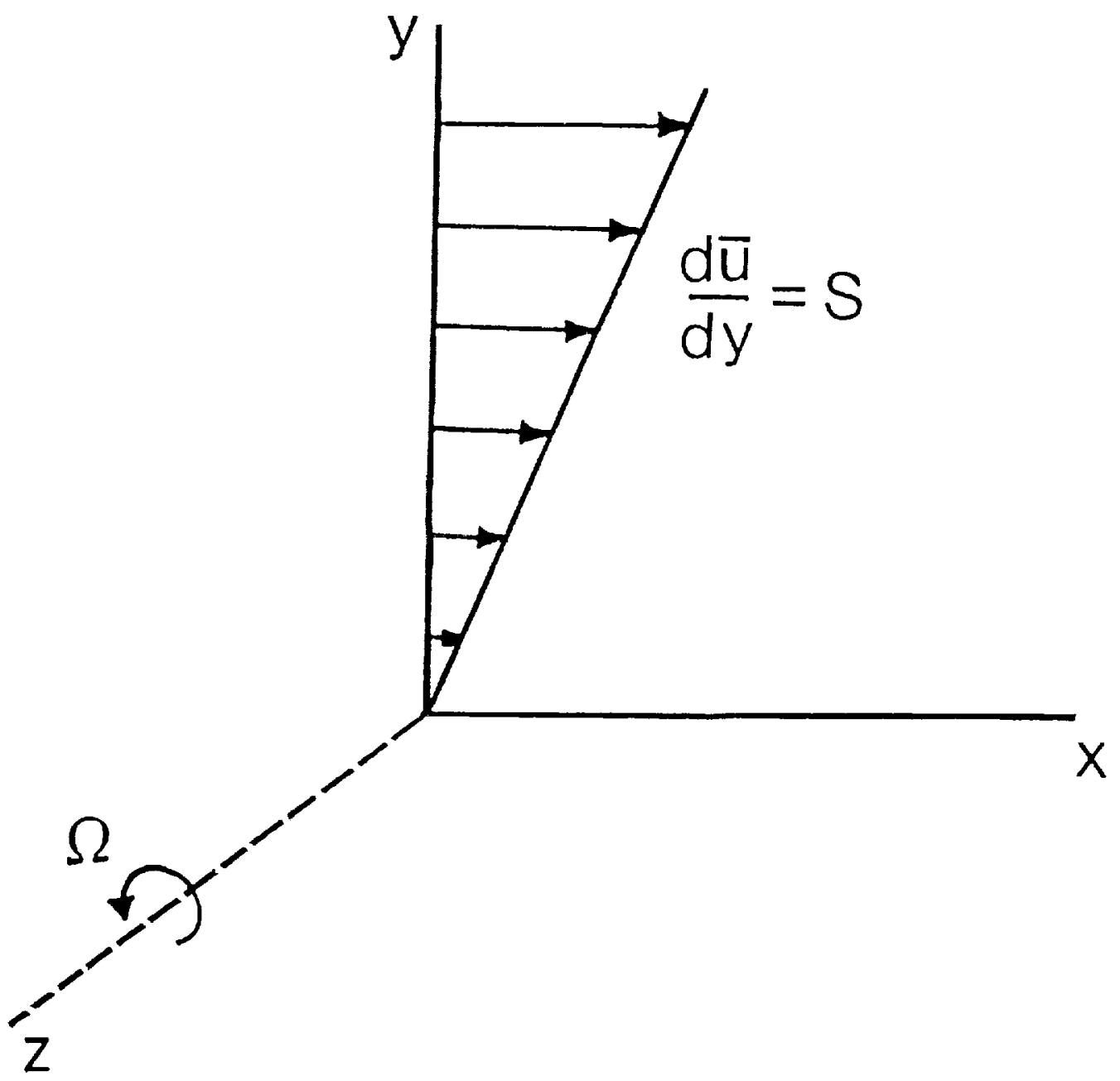

Figure 1. Schematic of homogeneous shear flow in a rolating frame. 


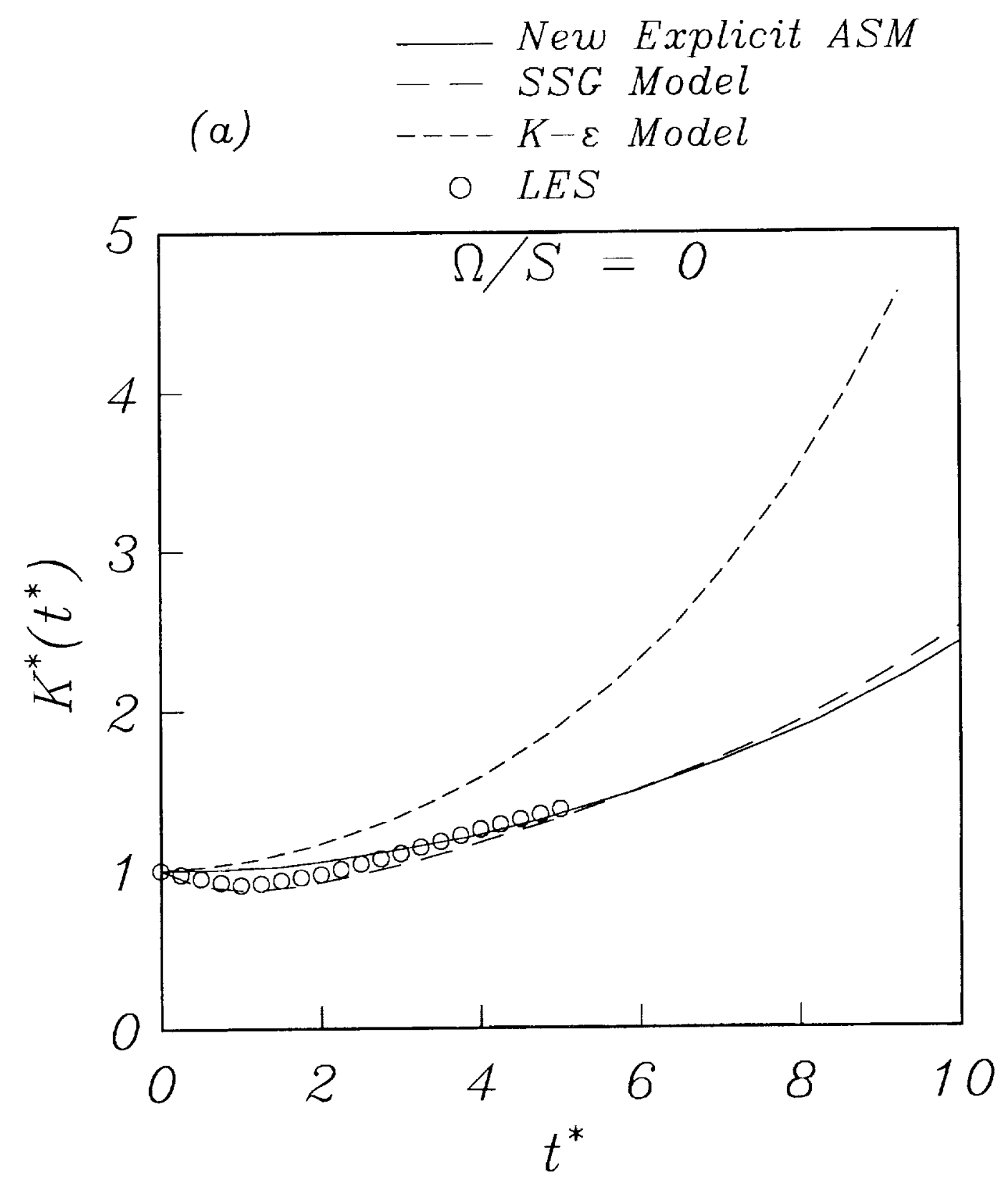

Figure 2. Time evolution of the turbulent kinetic energy in rotating homogencous shear flow: Comparison of the model predictions with the large-eddy simulations of Bardina ct al. (1983). (a) $\Omega / S=0$, (b) $\Omega / S=0.5$ and (c) $\Omega / S=-0.5$. 


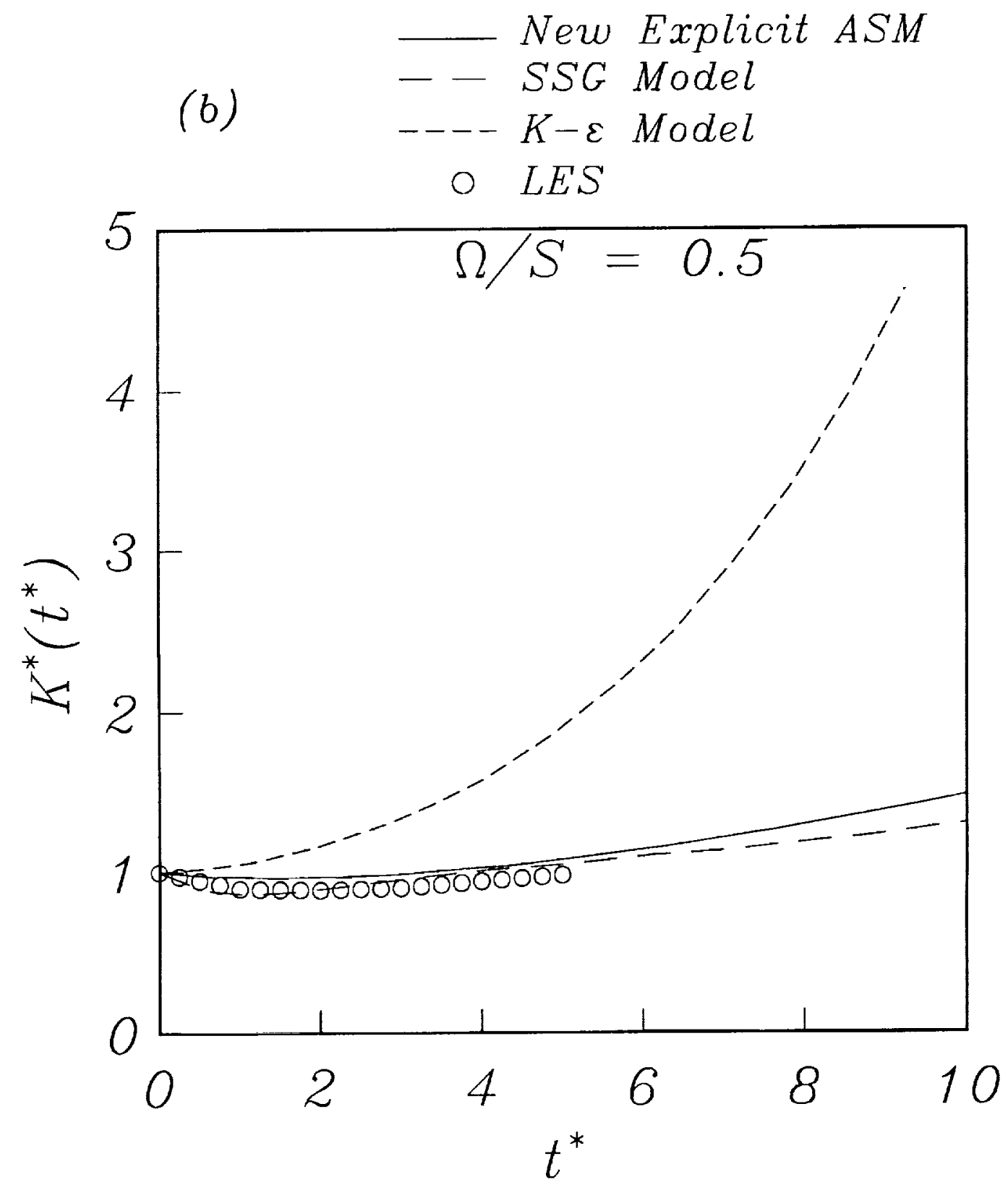

Figure 2. Time evolution of the turbulent kinetic energy in rotating homogeneous shear flow: Comparison of the model predictions with the large-eddy simulations of Bardina et al. (1983). (a) $\Omega / S=0$, (b) $\Omega / S=0.5$ and (c) $\Omega / S=-0.5$. 


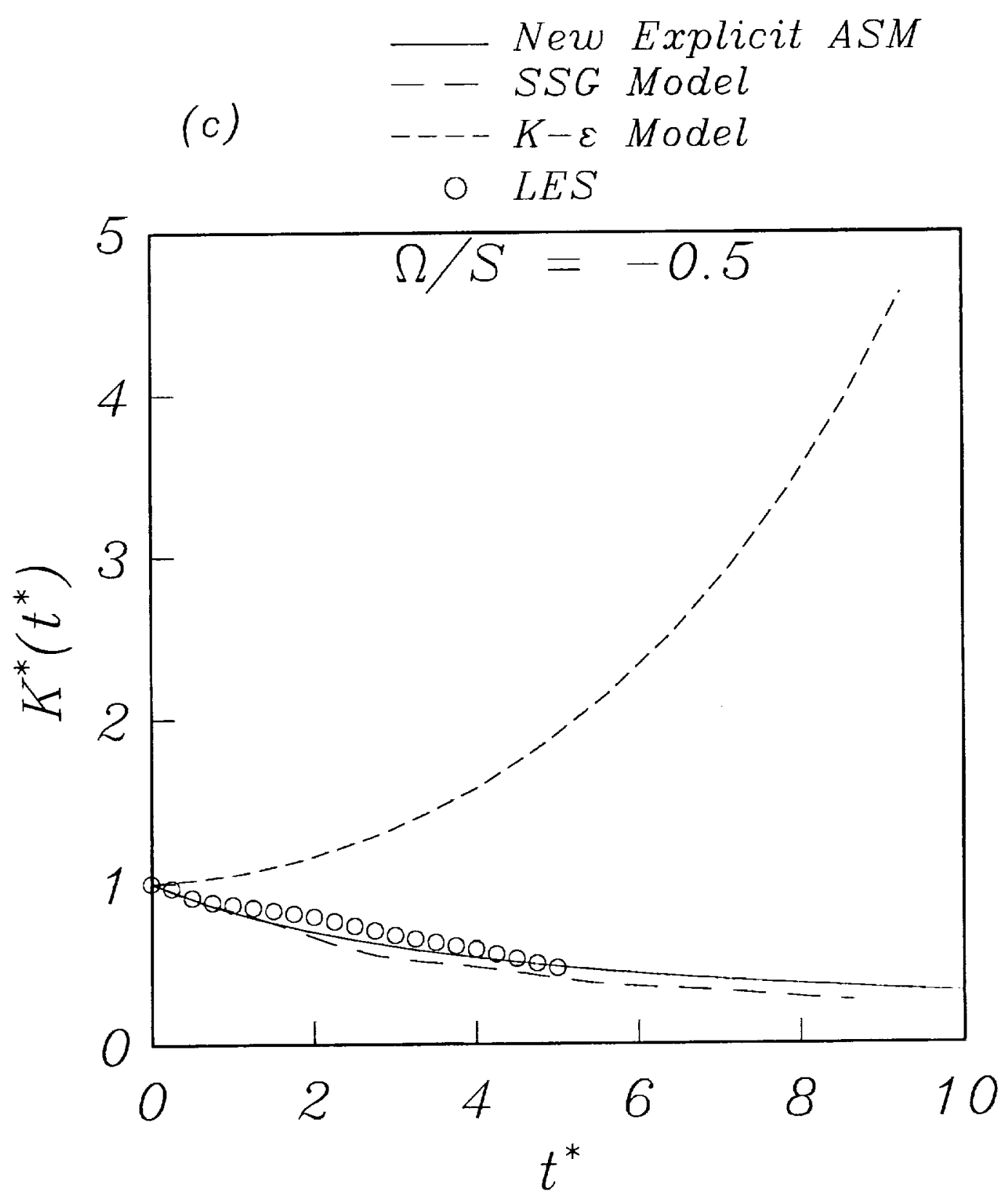

Figure 2. Time evolution of the turbulent kinetic energy in rolating homogencous shear flow: Comparison of the model predictions with the large-eddy simulations of Bardina ct al. (1983). (a) $\Omega / S=0$, (b) $\Omega / S=0.5$ and (c) $\Omega / S=-0.5$. 
(a) LES

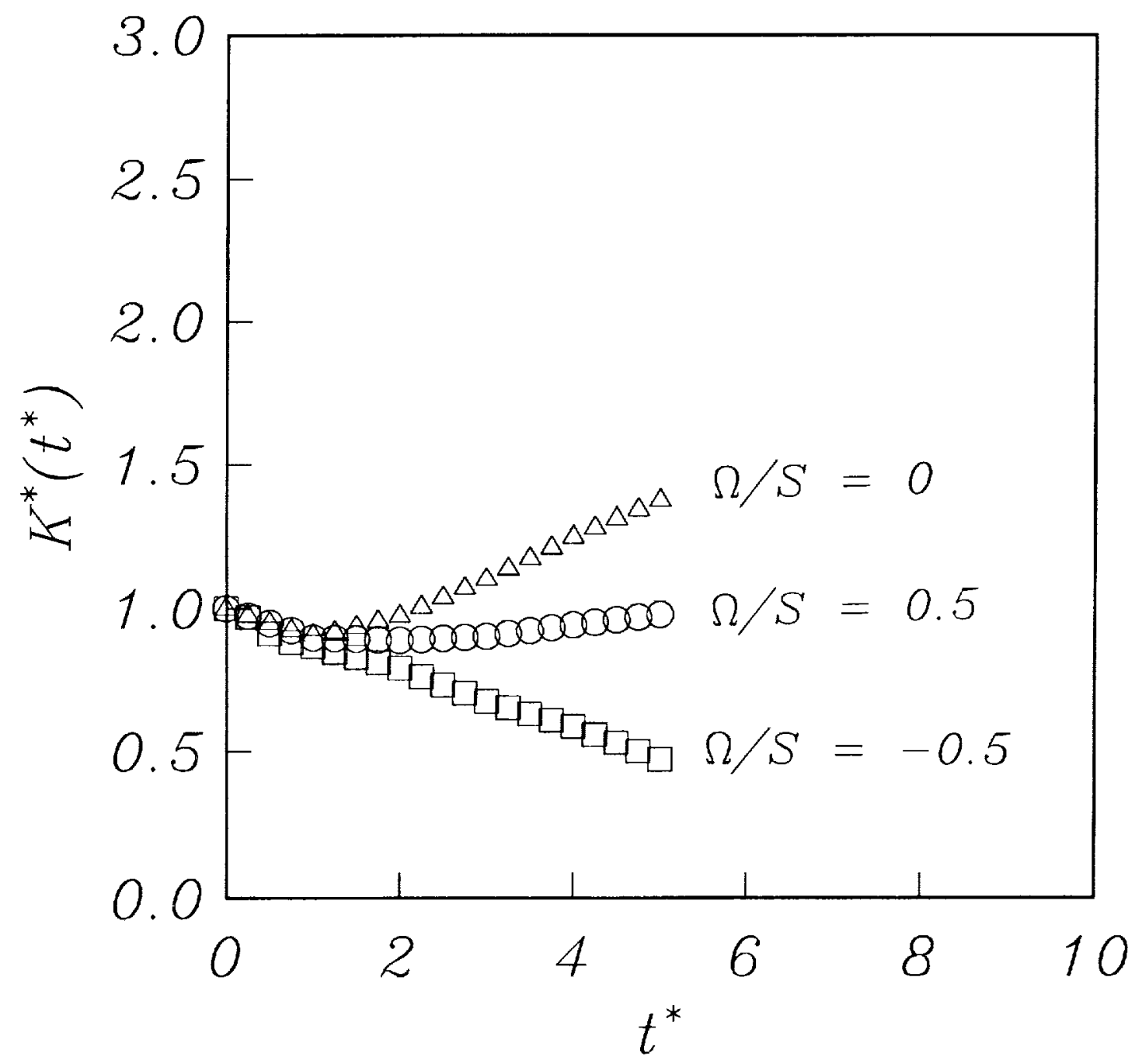

Figure 3. Time evolution of the turbulent kinetic energy in rotating homogeneous shear flow: (a) large-eddy simulations of Bardina et al. (1983), (b) new explicit algebraic stress model, and (c) standard $K-\varepsilon$ model. 
(b) New Explicit ASM

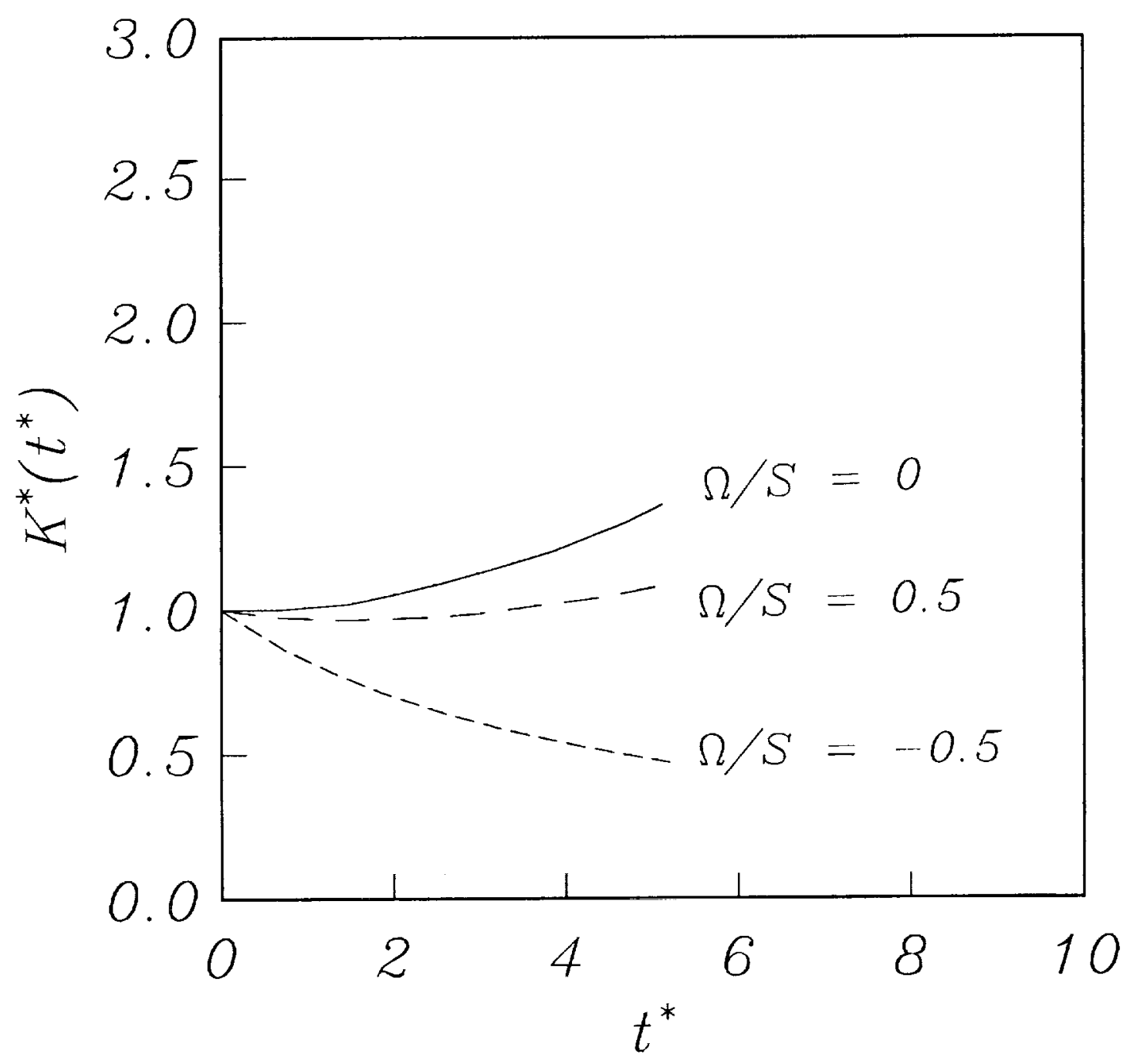

Figure 3. Time evolution of the turbulent kinetic energy in rotaling homogeneons shear flow: (a) large-eddy simulations of Bardina ct al. (1983), (b) new explicit algebraic stress model, and (c) standard $K-\varepsilon$ model. 
(c) $K-\varepsilon$ Model

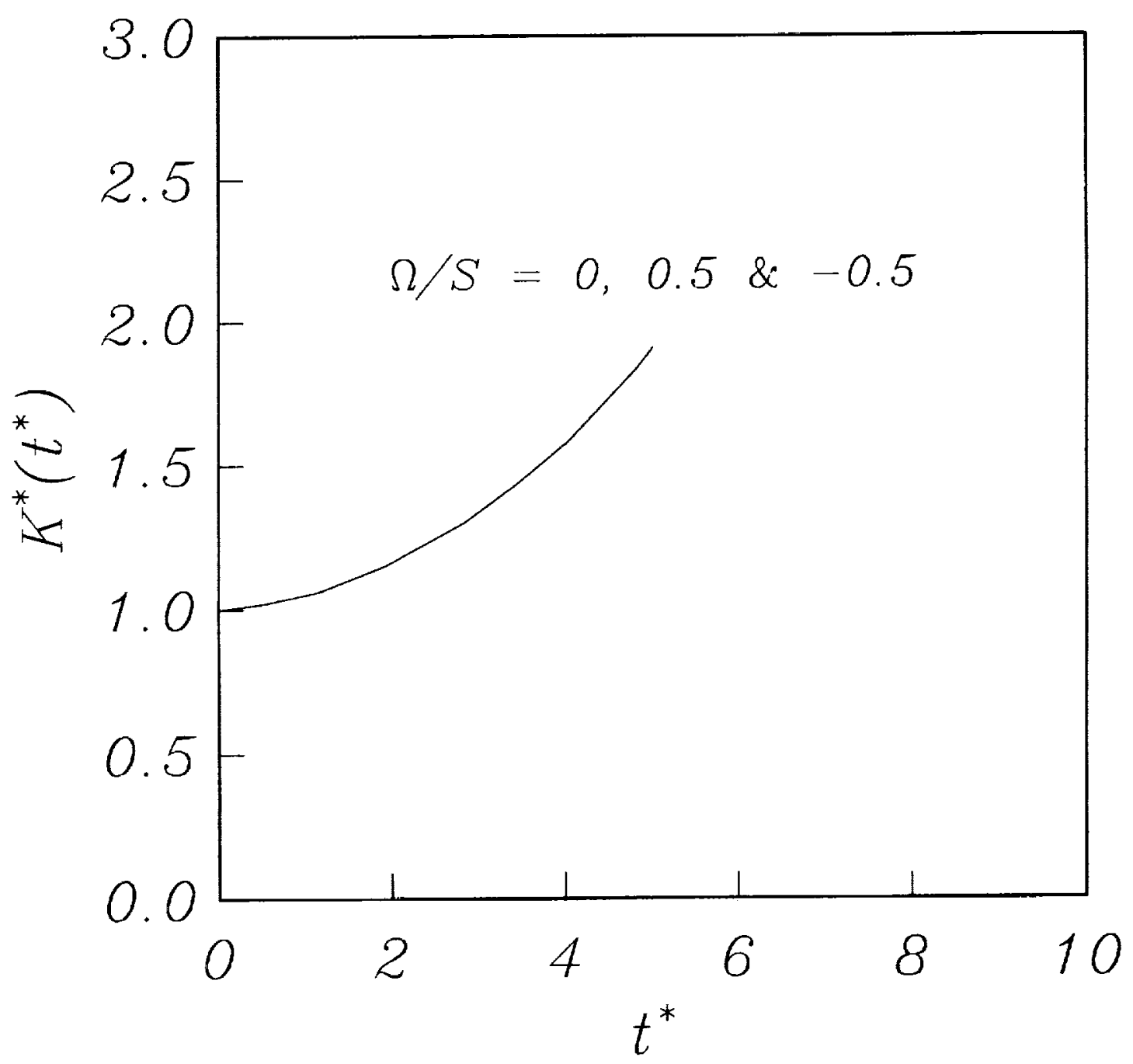

Figure 3. Tine cvolution of the turbulent kinetic energy in rotating homogeneons shear flow: (a) large-eddy simulations of Bardina et al. (1983), (b) new explicit algebraic stress model, and (c) standard $K-\varepsilon$ model. 


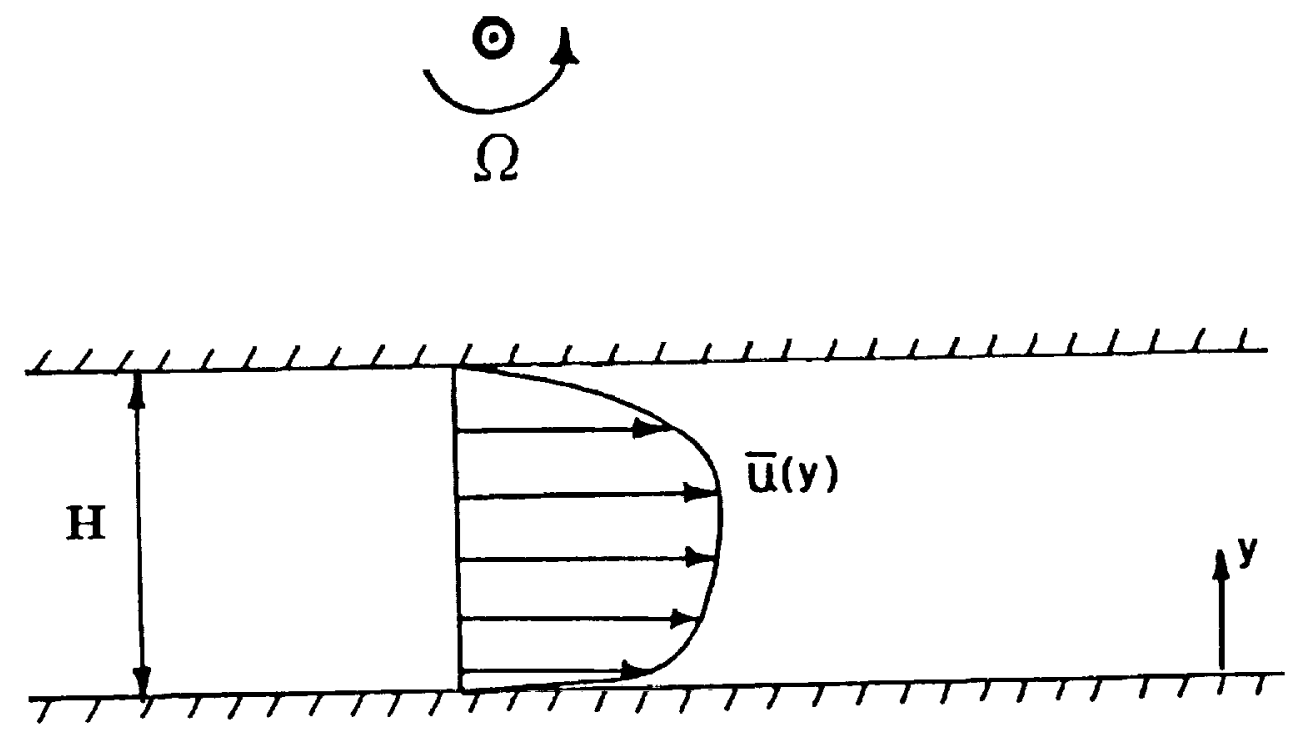

Figure 4. Schematic of fully-developed turbulent channel flow in a rotating frame. 


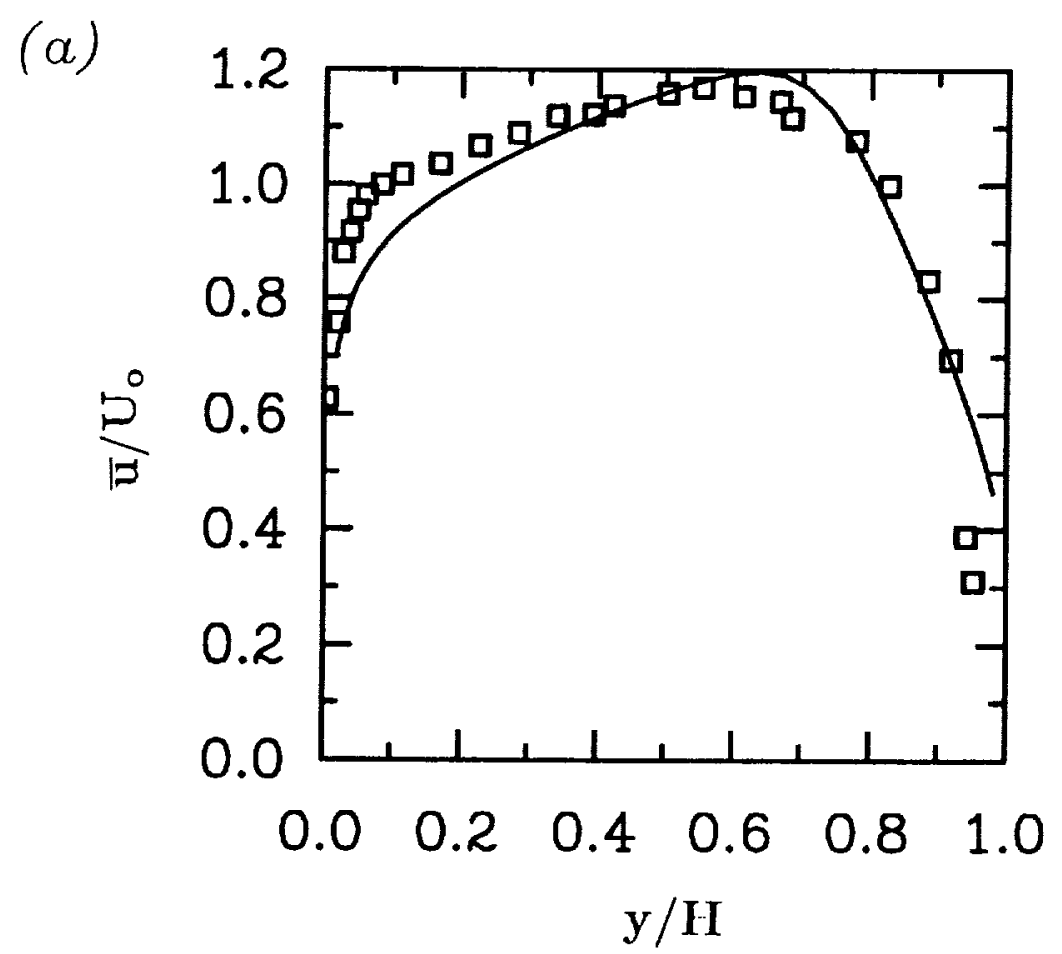

(b)

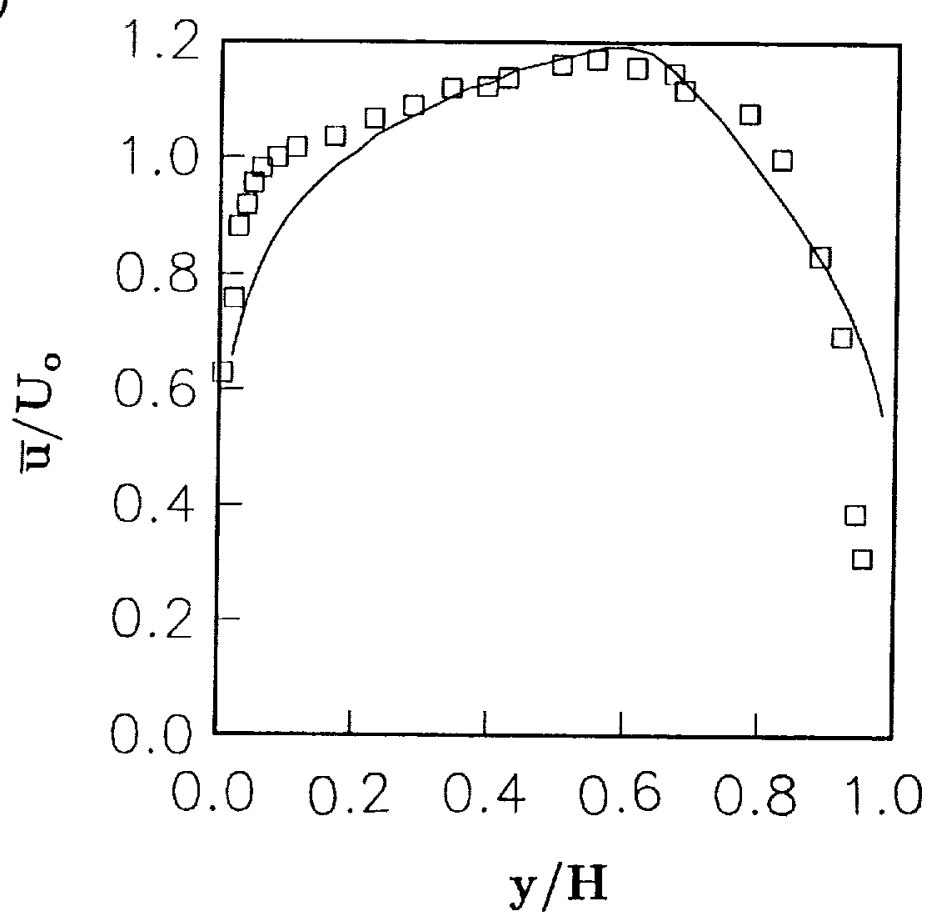

Figure 5. Comparison of the model predictions (-) for the mean velocity profile with the experimental data (D) of Johnston et al. (1972) for rotating channel flow $(R e=11,500, R o=$ 0.21): (a) SSG second-order closure (taken from Speziale et al. 1992) and (b) new explicit algebraic stress model. 
. 


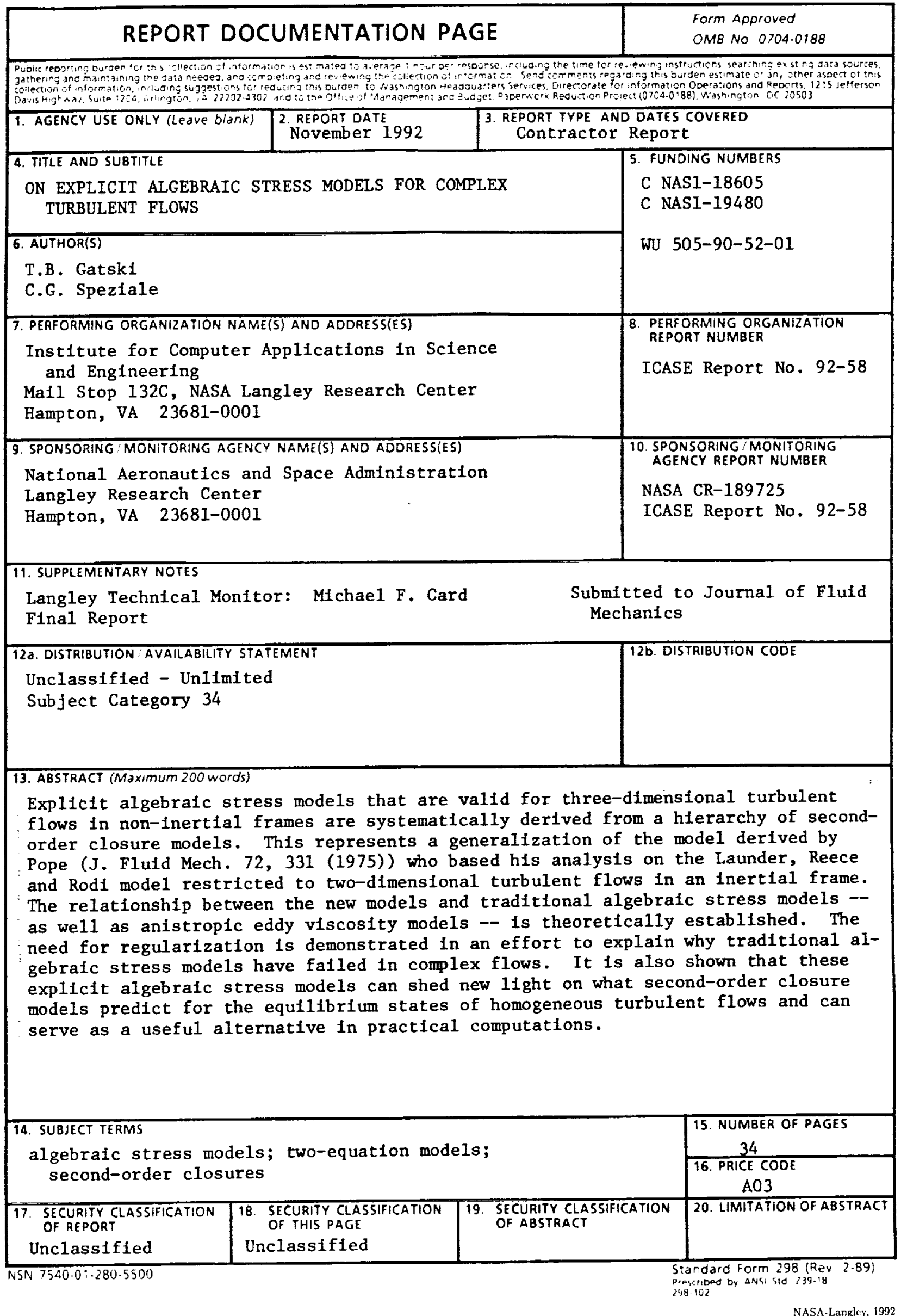

